\title{
THERMODYNAMICS OF THE HIGH-TEMPERATURE QUARK-GLUON PLASMA
}

\author{
Jean-Paul Blaizot and Edmond Iancu \\ Service de Physique Théorique, CEA/DSM-Saclay, \\ Unité de Recherche Associée au CNRS, 91191 Gif-sur-Yvette, France
}

Anton Rebhan

Institut für Theoretische Physik, Technische Universität Wien, Wiedner Hauptstr. 8-10, A-1040 Vienna, Austria

\begin{abstract}
We review the various methods which have been employed recently to describe the thermodynamics of the high temperature quark-gluon plasma using weak coupling techniques, and we compare their results with those of most recent lattice gauge calculations. Many of the difficulties encountered with perturbation theory at finite temperature are in fact not specific to QCD but are present in any field theory at finite temperature and will be discussed first in the simple example of the scalar field theory. We discuss the merits and limitations of various techniques which have been used to go beyond perturbation theory in the soft sector, such as dimensional reduction, screened perturbation theory or hard-thermal-loop perturbation theory, and $\Phi$-derivable approximations. In the last part of the review, we focus on the later, which lead to a remarkably simple expression for the entropy of the quark-gluon plasma. When complemented with further, physically motivated, approximations, this approach reproduces accurately the entropy obtained from lattice gauge calculations at temperatures above $2.5 T_{c}$, where $T_{c}$ is the deconfinement temperature. This calculation thus provides also support to the physical picture of the quark-gluon plasma as a gas of weakly interacting quasiparticles.
\end{abstract}




\section{Contents}

1 Introduction . . . . . . . . . . . . . . . . . . . 3

2 The scalar field theorv as a pedagogical example . . . . . . . . . . . . 8

$2.1 \quad$ Perturbation theorv and its difficulties . . . . . . . . . . . . . . 8

2.2 Order $q^{3}$ from various resummation schemes . . . . . . . . . . . . 13

2.2.1 The next-to-leading-order thermal mass . . . . . . . . . . 13

2.2.2 The plasmon effect in the pressure . . . . . . . . . . . . . 15

2.2 .3 Static resummation . . . . . . . . . . . . . . . . . . . . . . . . 15

2.2 .4 Dimensional reduction . . . . . . . . . . . . . . . 16

2.2.5 Screened perturbation theory . . . . . . . . . . . . . . 19

2.3 Self-consistent resummation . . . . . . . . . . . . . . 23

2.3.1 Skeleton expansion for thermodvnamical potential . . . . . 23

2.3.2 A simple model . . . . . . . . . . . . . . . . . . 24

2.3 .3 The entropv . . . . . . . . . . . . . . . . 29

2.3.4 Approximatelv self-consistent calculations of the entropv . 31

3 Quark-Gluon Plasma . . . . . . . . . . . . . . . . . . . 34

3.1 Perturbative results . . . . . . . . . . . . . . . . . . . . . . . . . . . . . . .

3.2 Lattice results . . . . . . . . . . . . . . . . . . . . . . . . 36

3.3 Dimensional reduction . . . . . . . . . . . . . . . . . . . . . . 37

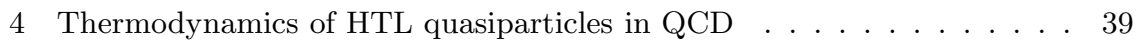

4.1 HTL-screened perturbation theorv . . . . . . . . . . . . . . . . . . 39

4.2 2PI formalism in gauge theories . . . . . . . . . . . . . . . . . . 42

4.3 Approximately self-consistent entropv and quark density . . . . . 43

4.3.1 HTL approximation . . . . . . . . . . . . . . . 44

4.3.2 Next-to-leading approximations . . . . . . . . . . . . . 48

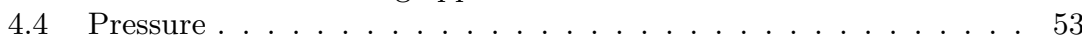

4.4.1 Finite chemical potential . . . . . . . . . . . . . . 54

4.5 Quark number susceptibilities . . . . . . . . . . . . . . 54

4.5.1 Diagonal quark number susceptibilities . . . . . . . . . . 55

4.5.2 Off-diagonal quark number susceptibilities . . . . . . . . . 56

5 Conclusion and perspectives . . . . . . . . . . . . . . . 58

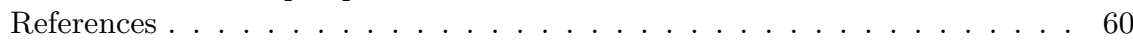




\section{Introduction}

Much of the motivation for studying heavy-ion collisions at ultra-relativistic energies is based on the expectation that matter at high temperature and/or density becomes simple: because of the asymptotic freedom of Quantum Chromodynamics (QCD), one expects that in this regime matter becomes a weakly interacting gas of quarks and gluons whose properties should be calculable in a weak coupling expansion.

The existence of weakly interacting quark matter was indeed anticipated on the basis of asymptotic freedom of $\mathrm{QCD}^{1}$. But the most compelling theoretical evidences for the existence of the quark-gluon plasma are coming from lattice gauge calculations (for recent reviews see e.g. Refs. ${ }^{23}$ ). These are at present the unique tools allowing a detailed study of the transition region where various interesting phenomena are taking place, such as colour deconfinement or chiral symmetry restoration. In this review, we shall not consider this transition region, ${ }^{\mathrm{a}}$, but focus rather on the high temperature phase, exploiting the fact that at sufficiently high temperature the effective gauge coupling constant should be small enough to allow for weak coupling calculations 5678 .

The physical picture which emerges from these weak coupling calculations is a simple one, and in many respect the quark-gluon plasma is very much like an ordinary electromagnetic plasma in the ultrarelativistic regime (with however specific effects related to the nonabelian gauge symmetry 91011). To zeroth order in an expansion in powers of the coupling $g$, the quark gluon plasma is a gas of noninteracting quarks and gluons, with typical momenta $k \sim T$. This is the ideal quark-gluon plasma. The interactions appear to alter only slightly this simple physical picture: they turn plasma particles into massive quasiparticles, and generate collective modes at small momenta. However, while the relevant degrees of freedom have been identified long ago, and effective theories to describe their dynamics are known 912131416, the calculation of the thermodynamics of the quark-gluon plasma using weak coupling techniques has remained unsuccessful until recently.

Strict expansions in power of the coupling constant have been pushed up to order $g^{5}$, both in QCD and in scalar field theories. In both cases, one observes a rather poor apparent convergence, the successive contributions oscillating wildly, unless the coupling is very small and the deviation from

$\overline{{ }^{\mathrm{a}} \text { For new ideas on how to cover this }}$ region by effective field theory methods see Refs. 4 . 
ideal-gas behaviour correspondingly minute. As we shall see, the difficulty may be related to the fact that what determines the accuracy of a weak coupling calculation in thermal field theories is not only the strength of the coupling, but also the magnitude of the thermal fluctuations. These vary according to the relevant momentum scales, so that the accuracy of the weak coupling expansion depends on which momentum scale contributes dominantly to the quantity under consideration.

To get orientation into these effects, let us then consider a massless scalar field theory with $g^{2} \phi^{4}$ interactions. In the noninteracting case the magnitude of the thermal fluctuations of the field is given by the simple formula (with $\varepsilon_{k}=k$ ):

$$
\left\langle\phi^{2}\right\rangle=\int \frac{\mathrm{d}^{3} k}{(2 \pi)^{3}} \frac{n\left(\varepsilon_{k}\right)}{\varepsilon_{k}}
$$

where $n\left(\varepsilon_{k}\right)=1 /\left(\mathrm{e}^{\beta \varepsilon_{k}}-1\right)$ is the Bose-Einstein distribution function. We shall use this formula as an approximation for the magnitude of the fluctuations also in the interacting case, eventually modifying $\varepsilon_{k}$ appropriately. In order to get a criterion for the expected validity of perturbation theory, we shall compare the expectation value of the "kinetic energy" $\left\langle(\partial \phi)^{2}\right\rangle$ with the "interaction energy" which we approximate as $g^{2}\left\langle\phi^{2}\right\rangle^{2}$.

For the plasma particles $\varepsilon_{k}=k \sim T$ and $\left\langle\phi^{2}\right\rangle_{T} \sim T^{2}$. Then indeed, when $g$ is small, the kinetic energy of a typical particle, $k^{2} \sim T^{2}$, is large compared to its interaction energy $\sim g^{2}\left\langle\phi^{2}\right\rangle_{T}$ : The short wavelength, or hard, fluctuations produce a small perturbation on the motion of a plasma particle. However, this is not so for an excitation at the momentum scale $k \sim g T$ : then the kinetic energy $k^{2} \sim(g T)^{2}$ is comparable to the contribution to the interaction energy coming from the coupling to the hard modes, $g^{2}\left\langle\phi^{2}\right\rangle_{T}^{2}$. Thus, the properties of an excitation with momentum $g T$ are expected to be nonperturbatively renormalized by the hard thermal fluctuations. And indeed, the scale $g T$ is that at which collective phenomena develop. The emergence of the Debye screening mass $m_{D} \sim g T$ in a plasma is one of the simplest examples of such phenomena. More generally, this renormalization of the soft mode dynamics by hard mode contributions invites a description in terms of an effective theory for the soft modes, the parameters of this effective theory being determined by the hard modes. The building blocks of such an effective theory have been dubbed hard thermal loops (HTL). In the scalar field theory, there is only one such HTL, which is a mass. But in QCD, there is an infinite number of them 910 .

Let us now consider the fluctuations at the soft scale $g T \ll T$. These 
fluctuations can be accurately described by classical fields. In fact the associated occupation numbers $n\left(\varepsilon_{k}\right)$ are large, and accordingly one can replace $n\left(\varepsilon_{k}\right)$ by $T / \varepsilon_{k}$ in eq. (1). Introducing an upper cut-off $g T$ in the momentum integral, one then gets:

$$
\left\langle\phi^{2}\right\rangle_{g T} \sim \int^{g T} \mathrm{~d}^{3} k \frac{T}{k^{2}} \sim g T^{2} .
$$

Thus $g^{2}\left\langle\phi^{2}\right\rangle_{g T} \sim g^{3} T^{2}$ is still of higher order than the kinetic term $g^{2} T^{2}$. In that sense the soft modes with $k \sim g T$ are still perturbative. Note however that they generate contributions to physical observables which are not analytic in $g^{2}$, as shown by the example of the order $g^{3}$ contribution to the energy density, which goes schematically as follows:

$$
\epsilon^{(3)} \sim \int_{0}^{g T} \mathrm{~d}^{3} k \varepsilon_{k} \frac{T}{\varepsilon_{k}} \sim T(g T)^{3} \sim g^{3} T^{4} .
$$

In fact, in contrast to the perturbation theory for the hard modes, the parameter which controls the perturbative expansion for the soft modes is $g$ instead of $g^{2}$ so that is it less accurate in general. As we shall see, much of the difficulties with the strict perturbative expansion can be attributed to the poor accuracy of perturbation theory in the soft sector, and the present review describes various solutions proposed to get around this problem.

The previous arguments concerning the fluctuations can be extended to QCD, where one should compare the kinetic energy $(\partial A)^{2}$ with the magnitude of the nonabelian interactions $g^{2} A^{4}$ (with $A$ the gauge potential). For the plasma particles $\varepsilon_{k}=k \sim T$ and $\left\langle A^{2}\right\rangle_{T} \sim T^{2}$. The associated electric (or magnetic) field fluctuations are $\left\langle E^{2}\right\rangle_{T} \sim\left\langle(\partial A)^{2}\right\rangle_{T} \sim k^{2}\left\langle A^{2}\right\rangle_{T} \sim T^{4}$ and are a dominant contribution to the plasma energy density. As already mentioned, these short wavelength, or hard, gauge field fluctuations produce a small perturbation on the motion of a plasma particle. However, this is not so for an excitation at the momentum scale $k \sim g T$ which is nonperturbatively renormalized by the hard thermal fluctuations. And again perturbation theory for the soft modes is governed by $g$, not $g^{2}$.

A new feature emerges however in QCD: at the ultrasoft momentum scale, $k \sim g^{2} T$, the unscreened magnetic fluctuations play a dominant role. At that scale it becomes necessary to distinguish the electric and the magnetic sectors (which provide comparable contributions at the scale $g T)$. The electric fluctuations decouple because the Debye screening mass $m_{D}^{2} \gg k^{2} \sim\left(g^{2} T\right)^{2}$ and their contribution is negligible, of order $g^{4} T^{2}$. However, because of the absence of static screening in the magnetic sector, 
we have there $\varepsilon_{k} \sim k$ and

$$
\left\langle A^{2}\right\rangle_{g^{2} T} \sim T \int_{0}^{g^{2} T} \mathrm{~d}^{3} k \frac{1}{k^{2}} \sim g^{2} T^{2},
$$

so that $(\partial A)^{2} \sim g^{6} T^{4} \sim g^{2}\left(A^{2}\right)^{2}$ : the fluctuations are no longer perturbative. This is the origin of the well-known breakdown of perturbation theory at order $g^{6}$.

Whereas one may argue that these non-perturbative magnetic fluctuations can contribute to the deviation of the pressure from the ideal gas result, as observed on the lattice, this contribution, being of order $g^{6}$, is presumably numerically rather small sufficiently above the transition temperature. At any rate, it is clear that the magnetic fluctuations have a priori no responsibility for the lack of convergence of the weak coupling expansion at lower orders (i.e., up to order $g^{5}$ ). This is further confirmed by the fact that a similar lack of convergence is seen also in other theories, like the scalar field theory. These magnetic fluctuations require genuine non-perturbative treatment, such as that based on a combination of dimensional reduction and lattice calculations 17. This will be briefly discussed. But most of the review will focus on purely analytical resummation techniques which have been proposed to improve perturbation theory in the soft sector.

Among those, the simplest one which attempts to account nonperturbatively for the dominant effect of interactions in the soft sector, which in the case of the scalar field is to generate a mass for the excitations, is the so-called "screened perturbation theory" 181920. This consists in a reorganization of the perturbative expansion, based on the following rewriting of the Lagrangian:

$$
\mathcal{L}=\mathcal{L}_{0}-\frac{1}{2} m^{2} \phi^{2}+\frac{1}{2} m^{2} \phi^{2}+\mathcal{L}_{i n t}=\mathcal{L}_{0}^{\prime}+\mathcal{L}_{\text {int }}^{\prime},
$$

with $\mathcal{L}_{0}^{\prime}=\mathcal{L}_{0}-(1 / 2) m^{2} \phi^{2}$. A perturbative expansion in terms of screened propagators (that is keeping the screening mass $m$ as a parameter, i.e. not as a perturbative correction to be expanded out) has been shown to be quite stable with good convergence properties. However, $m$ depends on the temperature, which makes the propagators explicitly temperature dependent, and the ultraviolet divergences which occur in high order calculations become temperature dependent. Thus, at any finite loop order, the ultraviolet renormalization gets artificially modified.

In the case of gauge theory, the effect of the interactions is more complicated than just generating a mass. But we know how to determine the dominant corrections to the self-energies. When the momenta are soft, these 
are given by the hard thermal loops 910 . By adding these corrections to the tree level Lagrangian, and subtracting them from the interaction part, one generates the so-called hard thermal loop perturbation theory (HTLPT) 21 .

$$
\mathcal{L}=\mathcal{L}_{0}+\mathcal{L}_{H T L}-\mathcal{L}_{H T L}+\mathcal{L}_{\text {int }}=\mathcal{L}_{0}^{\prime}+\mathcal{L}_{\text {int }}^{\prime} .
$$

The resulting perturbative expansion is made complicated however by the nonlocal nature of the hard thermal loop action, and by the necessity of introducing temperature dependent counter terms. Also, in such a scheme, one is led to use the hard thermal loop approximation in kinematical regimes where it is no longer a justifiable approximation.

$\Phi$-derivable approximations $\frac{22}{22}$ provide a more natural approach to propagator renormalization than screened perturbation theory, although the general goal is similar. It allows us to exploit fully the spectral information that one has about the plasma excitations. In particular the hard thermal loops enter as essential ingredients, ${ }^{232425}$ but in contrast to HTLPT, they contribute in an essential way only for external momenta where they are accurate. One of the main advantages of such approximations is that they lead to remarkable simplifications in the calculation of the entropy 26 This allows one, in particular, to bypass some of the difficulties of HTLPT. Besides, the entropy makes transparent the underlying physical picture of quasiparticles, which (in simpler models) has been shown to provide rather accurate fits to lattice data $27 / 28 / 2930$. The quasiparticle picture assumes that the dominant effect of the interactions can be incorporated in the spectral properties of suitably defined quasiparticles with small residual interactions; this is precisely what comes out of the entropy calculation.

Finally, we should mention other attempts to improve the behaviour of perturbation theory by using various mathematical extrapolation schemes. Thus, extrapolations have been constructed, based on the first terms of

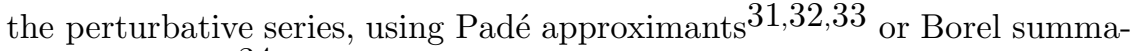
tion techniques 34 . The resulting expressions are indeed smooth functions of the coupling, better behaved than polynomial approximations truncated at order $g^{5}$ or lower, with a weak dependence on the renormalization scale. However these techniques, which offer little physical insight, will not be discussed further here.

The outline of the paper is as follows. In the next section, we shall review various features of perturbation theory in quantum field theory at finite temperature, using the simple example of the scalar field. This will offer us the possibility to illustrate most of the difficulties encountered in thermal gauge theories, and also the various resummation schemes which have been 
proposed in the literature. Section 3 presents a brief summary of the state of the art concerning the perturbative calculations in QCD, those based on dimensional reduction, as well as recent lattice data. In section 4 we describe the calculation of the entropy and emphasize its technical simplicity and its physical content. The last section of the review summarizes the various conclusions and puts the different approaches into a general perspective.

\section{The scalar field theory as a pedagogical example}

As mentioned in the introduction, many of the difficulties of weak coupling calculations at high temperature are not specific to gauge theories. They will be illustrated in this section by studying the thermodynamics of a scalar field with Lagrangian

$$
\mathcal{L}=\frac{1}{2} \partial_{\mu} \phi \partial^{\mu} \phi-\frac{m^{2}}{2} \phi^{2}-\frac{\lambda}{4 !} \phi^{4} .
$$

We shall often use $g^{2} \equiv \lambda / 4$ ! as an alternative notation for the coupling strength.

\subsection{Perturbation theory and its difficulties}

The weak-coupling expansion of the pressure in the case $m=0$ has been computed to order $\lambda^{5 / 2}$ (or $g^{5}$ ), and reads 35136137 .

$$
\begin{aligned}
P=P_{0} & {\left[1-\frac{15}{8}\left(\frac{g}{\pi}\right)^{2}+\frac{15}{2}\left(\frac{g}{\pi}\right)^{3}+\frac{135}{16}\left(\log \frac{\bar{\mu}}{2 \pi T}+0.4046\right)\left(\frac{g}{\pi}\right)^{4}\right.} \\
& \left.-\frac{405}{8}\left(\log \frac{\bar{\mu}}{2 \pi T}-\frac{4}{3} \log \frac{g}{\pi}-0.9908\right)\left(\frac{g}{\pi}\right)^{5}+\mathcal{O}\left(g^{6} \log g\right)\right]
\end{aligned}
$$

where $P_{0}=\left(\pi^{2} / 90\right) T^{4}$ is the pressure of an ideal gas of free massless bosons, and $g^{2}(\bar{\mu}) \equiv \lambda(\bar{\mu}) / 24$ is the $\overline{\mathrm{MS}}$ coupling constant at the renormalization scale $\bar{\mu}$. This formula calls for several remarks.

First we note that, to order $\lambda^{5 / 2}, P$ is formally independent of $\bar{\mu}$ (in the sense that $d P / d \bar{\mu}$ involves terms of order $\lambda^{3}$ at least). This is easily verified using the renormalization group equation satisfied by the renormalized coupling $\lambda(\bar{\mu})$ :

$$
\bar{\mu} \frac{d \lambda}{d \bar{\mu}}=3 \frac{\lambda^{2}}{16 \pi^{2}}+\mathcal{O}\left(\lambda^{3}\right) .
$$

But the approximate expression (8) for $P$ does depend numerically on $\bar{\mu}$. In order to avoid large logarithms $\sim \ln (\bar{\mu} / 2 \pi T)$ in the expansion (8) one 


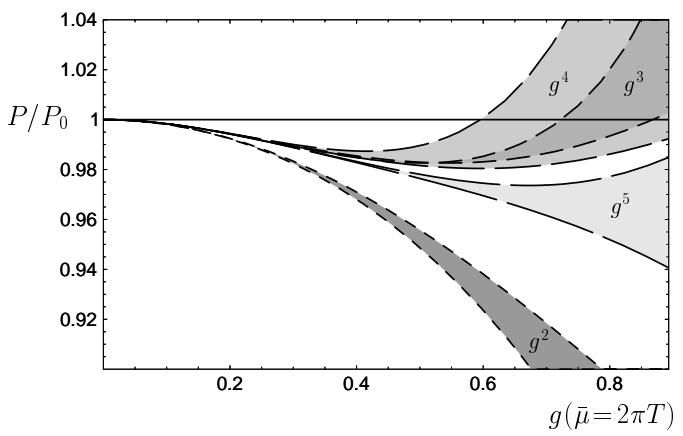

Fig. 1. Weak-coupling expansion through orders $g^{2}, g^{3}, g^{4}$, and $g^{5}$ for the pressure normalized to that of an ideal gas as a function of $g(2 \pi T)$ in $\phi^{4}$ theory.

may choose $\bar{\mu} \simeq 2 \pi T$. With this choice $\lambda(\bar{\mu})$ becomes effectively a function of the temperature. In the case of $\mathrm{QCD}$ where the $\beta$-function is negative, this leads to the expectation that the effective coupling becomes small at large temperature.

In Fig. 1] we show the successive perturbative approximations to $P / P_{0}$ as a function of $g(2 \pi T)$. Each partial sum is shown as a band obtained by varying $\bar{\mu}$ from $\pi T$ to $4 \pi T$, which gives an indication of the theoretical uncertainty in the calculation. Thus, in drawing this plot, $g(\bar{\mu})$ has been calculated from $g(2 \pi T)$ by using the renormalization group equation (9). The lack of convergence of the weak-coupling expansion is evident in Fig. [1 We can infer from Fig. 1 that the expansion in powers of $g$ ceases to make sense as soon as $g(2 \pi T) \gtrsim 0.4$.

Another remark concerns the presence of terms non-analytic in the coupling constant $\lambda$, i.e., terms of order $g^{3} \sim \lambda^{3 / 2}, g^{5} \sim \lambda^{5 / 2}$, and also terms involving $\ln g$, in the perturbative expansion in eq. (8). As we shall see shortly, these terms result from infinite resummations of subsets of diagrams of perturbation theory.

At this stage, it is instructive to compare with the corresponding expansion of the screening "Debye" mass $m_{D}$, defined from the the static propagator by ${ }^{\mathrm{b}}$

$$
\mathbf{p}^{2}+m_{D}^{2}+\Pi(0, \mathbf{p})=0 \quad \text { at } \mathbf{p}^{2}=-m_{D}^{2},
$$

\footnotetext{
${ }^{\mathrm{b}} \mathrm{A}$ definition through the location of the pole rather than the infrared limit of the self-energy is crucial to make $m_{D}$ gauge-independent in non-Abelian theories 38 , this definition is also renormalization-group invariant 3940 .
} 


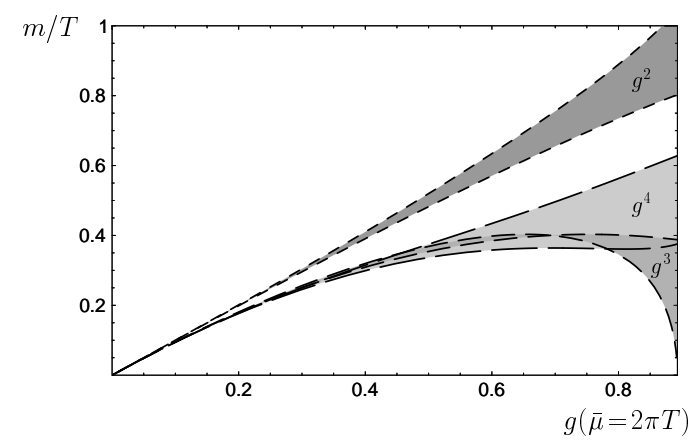

Fig. 2. Weak-coupling expansion to orders $g^{2}, g^{3}$, and $g^{4}$ for the screening mass normalized to the temperature as a function of $g(2 \pi T)$.

where $\Pi\left(p_{0}, \mathbf{p}\right)$ is the self-energy. For the massless case $(m=0)$, the weak coupling expansion of $m_{D}^{2}$ is known to order $g^{4}$, and reads 37 .

$$
m_{D}^{2}=g^{2} T^{2}\left\{1-3 \frac{g}{\pi}-\frac{9}{2}\left[\ln \frac{\bar{\mu}}{2 \pi T}-\frac{4}{3} \ln \frac{g}{\pi}-2.415\right]\left(\frac{g}{\pi}\right)^{2}+\mathcal{O}\left(g^{3}\right)\right\} .
$$

Note that, with our notation $g^{2} \equiv \lambda / 24$ for the coupling constant, the leading-order contribution in eq. (11) is simply

$$
\hat{m}_{D}=g T,
$$

which happens to be the same relation as in pure-glue QCD to leading order, cf. (91). As for the pressure, non-analytic terms $\left(g^{3}, \ln 1 / g\right)$ occur in the expression of $m_{D}^{2}$.

In Fig. 21 we show the screening mass $m_{D}$ normalized to the leading order result $\hat{m}_{D}$ as a function of $g(2 \pi T)$, for each of the three successive

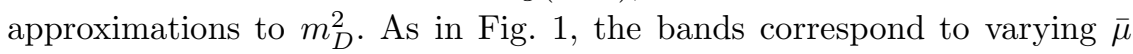
from $\pi T$ to $4 \pi T$. The poor convergence is again evident, with a pattern similar to that in Fig. 1 a large difference between the order- $g^{2}$ and order- $g^{3}$ approximations, and a larger sensitivity to the value of the renormalization scale for the order $g^{4}$.

In order to get some insight on this peculiar behaviour of the expansions (8) and (11), we shall briefly recall how the various terms are obtained, up to order $g^{3}$.

We start with the zeroth order calculation. We have $P_{0}=-\Omega_{0} / V$, with

$$
\frac{\Omega_{0}}{V}=\frac{1}{2} T \sum_{n} \int \frac{\mathrm{d}^{3} k}{(2 \pi)^{3}} \ln \left(\omega_{n}^{2}+\mathbf{k}^{2}+m^{2}\right)
$$




$$
=\frac{1}{2} \int \frac{d^{3} k}{(2 \pi)^{3}} \varepsilon_{k}+T \int \frac{d^{3} k}{(2 \pi)^{3}} \log \left(1-\mathrm{e}^{-\varepsilon_{k} / T}\right),
$$

where $\omega_{n}=2 n \pi T$ is a bosonic Matsubara frequency and $\varepsilon_{k}^{2}=k^{2}+m^{2}$. The first term in the second line of eq. (13) is the sum of the 'zero-point' energies of free massive bosons. This is ultraviolet divergent, but independent of the temperature; it can therefore be absorbed in the redefinition of the vacuum energy, and discarded. The second term is temperature-dependent and finite; this is recognized as minus the pressure of the ideal gas of scalar particles. For $m \ll T$ this can be expanded as:

$$
P_{0}(m)=-T \int \frac{d^{3} k}{(2 \pi)^{3}} \ln \left(1-e^{-\beta \varepsilon_{k}}\right)=\frac{\pi^{2} T^{4}}{90}-\frac{m^{2} T^{2}}{24}+\frac{m^{3} T}{12 \pi}+\ldots,
$$

where the neglected terms start at order $m^{4} \ln (m / T)$.

Consider now the contributions of order $g^{2}$ to the self-energy and the thermodynamic potential, denoted as $\Pi_{2}$ and $\Omega_{2}$, respectively. $\Pi_{2}$ is given by the "tadpole" diagram of Fig. 3. This gives:

$$
\begin{aligned}
\Pi_{2} & =\frac{\lambda}{2} T \sum_{n} \int \frac{\mathrm{d}^{3} k}{(2 \pi)^{3}} \frac{1}{\omega_{n}^{2}+\mathbf{k}^{2}+m^{2}} \equiv \frac{\lambda}{2} I(m) \\
& =\frac{\lambda}{2} \int \frac{d^{3} k}{(2 \pi)^{3}} \frac{1+2 n\left(\varepsilon_{k}\right)}{2 \varepsilon_{k}} \equiv \frac{\lambda}{2}\left[I_{0}(m)+I_{T}(m)\right],
\end{aligned}
$$

where $n(\omega)$ is the Bose-Einstein thermal distribution:

$$
n(\omega)=\frac{1}{\mathrm{e}^{\beta \omega}-1},
$$

and the notation $I(m)$ for the sum-integral, as well as $I_{0}(m)$ and $I_{T}(m)$ for its zero temperature and thermal contributions, will be used repeatedly in the following. The first term in the second line of eq. (15) is ultraviolet divergent, but independent of the temperature, so it can be absorbed into the definition of the (zero-temperature) mass. For instance, is we choose the mass parameter $m$ in the Lagrangian to be the physical mass at $T=0$, we must add a counterterm $\frac{1}{2} \delta m^{2} \phi^{2}$ in the Lagrangian, with $\delta m^{2}$ chosen

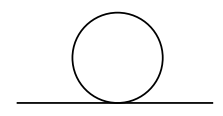

Fig. 3. Lowest order correction to the self-energy in scalar $\phi^{4}$ theory. 


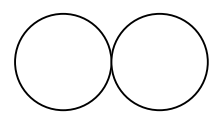

Fig. 4. The lowest order correction to the thermodynamic potential in $\phi^{4}$ theory.

so that:

$$
\frac{\lambda}{2} I_{0}(m)+\delta m^{2}=0
$$

Then, the lowest-order self-energy correction due to the thermal fluctuations reads:

$$
\Pi_{2}=\frac{\lambda}{2} I_{T}(m) \approx \frac{\lambda}{2}\left\{\frac{T^{2}}{12}-\frac{m T}{4 \pi}-\frac{m^{2}}{16 \pi^{2}}\left(\ln \frac{m^{2}}{(4 \pi T)^{2}}+2 \gamma-1\right)\right\},
$$

where the expansion in the r.h.s. holds when $m \ll T$. In particular, when $m=0$, we recover the result $\Pi_{2}=\hat{m}_{D}^{2}$, cf. eqs. (11)-(12). This quantity is generally dubbed a "hard thermal loop" (HTL), because when $m \ll T$ the dominant momenta in the one-loop integral (18) are hard $(k \sim T)$.

The contribution $\Omega_{2}$ is given by the 2 -loop diagram in Fig. 4 and reads:

$$
\frac{\Omega_{2}}{V}=\frac{\lambda}{4 !}\left\langle\phi^{4}\right\rangle_{0}+\frac{1}{2} \delta m^{2}\left\langle\phi^{2}\right\rangle_{0}=\frac{\lambda}{8}\left(I_{T}^{2}(m)-I_{0}^{2}(m)\right),
$$

where we have also included the contribution of the mass counterterm and used eq. (17) to verify that the temperature-dependent ultraviolet divergences cancel out, as they should. The second term in the last line in eq. (19) is divergent, but contributes only to the vacuum energy density, so that it can be discarded. For $m=0$, eq. (19) yields:

$$
P_{2}=-\frac{\lambda}{1152} T^{4}=-\frac{15}{8}\left(\frac{g}{\pi}\right)^{2} P_{0},
$$

in agreement with eq. [8].

At the next order in the loop expansion, the self-energy receives contributions from the 2-loop diagrams. However, some of these contributions are infrared divergent in the massless limit $m=0$. Consider, e.g., the second diagram displayed in Fig. [5] The upper loop (together with the corresponding mass counterterm, not shown explicitly in Fig. (5) is recognized as the "hard thermal loop" computed previously. Thus, this particular 2-loop contribution reads:

$$
\Pi_{2 L}^{(a)}=-\frac{\lambda}{2} T \sum_{n} \int \frac{\mathrm{d}^{3} k}{(2 \pi)^{3}} \frac{\hat{m}_{D}^{2}}{\left(\omega_{n}^{2}+k^{2}\right)^{2}} .
$$


The three-dimensional integral over $k$ in eq. (21) has a linear infrared divergence, coming from the term with $\omega_{n}=0$. (For the other terms, $\omega_{n} \neq 0$, the Matsubara frequency $\omega_{n}$ acts effectively as an infrared cutoff.) A non-zero mass $m$ would cut off this divergence, and give a contribution $\sim \lambda \hat{m}_{D}^{2}(T / m) \sim g^{4} T^{3} / m$. As we shall see, when $m \ll g T$, the real cutoff is not actually the mass $m$ itself, but the "thermal mass" $\hat{m}_{D}=g T$ induced by the thermal fluctuations. Then $\Pi_{2 L}^{(a)} \sim g^{4}\left(T^{3} / \hat{m}_{D}\right) \sim g^{3} T^{2}$. This illustrates how nonanalytic terms arise in eqs. (8) and (11): potential infrared divergences are cut-off by 'thermal masses' which, through their dependence on $g$, modify the original expansion in powers of the coupling constant. We discuss this in more detail in the next subsection.

\subsection{Order $g^{3}$ from various resummation schemes}

\subsubsection{The next-to-leading-order thermal mass}

The effect of a thermal mass is most directly seen by carrying out perturbative calculations with the dressed propagator

$$
\hat{D}\left(i \omega_{n}, \mathbf{k}\right) \equiv \frac{1}{\omega_{n}^{2}+\mathbf{k}^{2}+\hat{m}_{D}^{2}} .
$$

For the calculation of the tadpole, this corresponds to resumming the diagrams of bare perturbation theory shown in Fig. 5 (each of the inserted tadpoles in Fig. [5] is accompanied by a mass counterterm which removes its ultraviolet divergence in the vacuum, cf. eq. (17)).
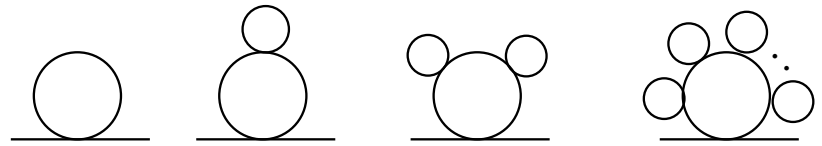

Fig. 5. Diagrams that are resummed when computing the tadpole in Fig. 3 with the dressed propagator in eq. 22.

Since $\hat{m}_{D}=g T$, this resummation involves arbitrarily high powers of g. However, the final result can be read from eq. (18) in which we set $m=\hat{m}_{D}=g T$ :

$$
\Pi_{\text {tadpole }}=g^{2} T^{2}-\frac{3}{\pi} g^{3} T^{2}+\mathcal{O}\left(g^{4} \ln g\right) .
$$

This formula exhibits the next-to-leading order contribution to the thermal mass, of order $g^{3}$, consistent with eq. (11). This order- $g^{3}$ contribution comes 
from the term linear in $m$ in the r.h.s. of eq. (18). It thus is related to the non-analyticity of the thermal integral $I_{T}(m)$ as a function of $m^{2}$ and to the infrared divergences which occur when one attempts to evaluate this integral after expanding the integrand in powers of $\mathrm{m}^{2}$. These infrared divergences are easily identified in the sum of the diagrams in Fig. 5 with at least two loops:

$$
\begin{aligned}
\Pi_{\text {ring }} & =\frac{\lambda}{2} T \sum_{n} \int \frac{\mathrm{d}^{3} k}{(2 \pi)^{3}}\left\{\frac{1}{\omega_{n}^{2}+k^{2}+\hat{m}_{D}^{2}}-\frac{1}{\omega_{n}^{2}+k^{2}}\right\} \\
& =12 g^{2} T \sum_{\omega_{n}} \int \frac{\mathrm{d}^{3} k}{(2 \pi)^{3}} \frac{1}{\omega_{n}^{2}+k^{2}} \sum_{l \geq 1}\left(\frac{-\hat{m}_{D}^{2}}{\omega_{n}^{2}+k^{2}}\right)^{l},
\end{aligned}
$$

where the subtracted term is the massless tadpole. The term with $l=1$ corresponds to the 2-loop diagram that we have considered separately in eq. (21). Each of the terms with $\omega_{n}^{2}>0$ in eq. (24) starts to contribute at order $g^{2} \hat{m}_{D}^{2} \sim g^{4}$. But the terms with $\omega_{n}=0$ are individually infrared divergent for any $l$, and the divergences cancel only in the sum of all the terms. In fact, the contribution of order $g^{3}$ arises from the static modes alone, i.e., the modes with zero Matsubara frequency. If we separate their contribution in eq. (24), we obtain indeed

$$
\Pi_{\text {ring }}^{(3)}=12 g^{2} T \int \frac{\mathrm{d}^{3} k}{(2 \pi)^{3}}\left\{\frac{1}{k^{2}+\hat{m}_{D}^{2}}-\frac{1}{k^{2}}\right\}=-\frac{3}{\pi} g^{3} T^{2},
$$

where we recognize the order $g^{3}$ contribution in eq. (23).

It is instructive to look at this contribution from another perspective. As we have argued before, the dominant contribution to $I_{T}(m)$ in eq. (18) comes from momenta $k \sim T$. The subleading contribution is coming from soft momenta $k \ll T$. To isolate it, we subtract the hard modes contribution, which amounts to subtract $I_{T}(0)$ from $I_{T}(m)$ in eq. (18), and write:

$$
I_{T}(m)-I_{T}(0) \simeq T \int \frac{d^{3} k}{(2 \pi)^{3}}\left(\frac{1}{k^{2}+\hat{m}_{D}^{2}}-\frac{1}{k^{2}}\right)=-\frac{\hat{m}_{D} T}{4 \pi} .
$$

After multiplication by $\lambda / 2=12 g^{2}$, one recognizes the $g^{3}$ term of eq. (23). In writing the approximate equality above, we have used the fact that the dominant contribution in the integral (26) comes from momenta $k \ll T$, so that we can use

$$
n\left(\varepsilon_{k}\right) \approx \frac{T}{\varepsilon_{k}} \quad \text { for } \quad \varepsilon_{k} \ll T .
$$

Note that the above resummation of the thermal mass, focusing on the static mode, hides an ultraviolet problem that we have ignored so far. 
Namely, in order to obtain the finite result in eq. (23), we had to cancel an ultraviolet divergence, cf. eq. (17), which now involves the thermal mass, and is therefore temperature dependent. The $T$-dependent divergent piece is of order $g^{4}$ however (since proportional to $g^{2} \hat{m}_{D}^{2}$ ), and thus does not formally affect the order $-g^{3}$ calculation. But it signals that our calculation is not complete. The divergence is identified in eq.(24) in the term with $l=1$, i.e., in the 2-loop diagram in Fig. [5 after performing the Matsubara sum. The overall divergence of the 2-loop diagram arises as the product of a vacuum divergence in the 4-point function subgraph (the lower loop) times the finite-temperature piece of the upper loop. Thus, the divergence is removed by the vacuum renormalization of the 4 -point function, i.e., by adding a 1-loop counterterm to the vertex in the tadpole.

\subsubsection{The plasmon effect in the pressure}
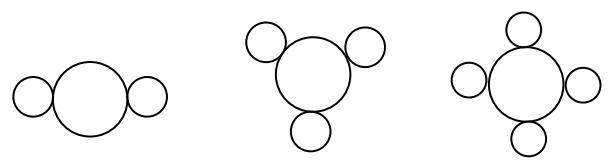

Fig. 6. The "ring" diagrams responsible for the order $-g^{3}$ effect in the pressure.

The contribution of order $g^{3}$ to the pressure, also known as the "plasmon effect", comes again from resumming diagrams which are infrared divergent in the naïve diagrammatic expansion. These are the "ring" diagrams in Fig. [6] in which the central loop is static $\left(\omega_{n}=0\right)$, while each outer loop represents an insertion of $\hat{m}_{D}^{2}$. This series can be easily summed up, with an infrared finite result $\underline{5}$.

$$
\begin{aligned}
P_{3} & =-\frac{T}{2} \int \frac{\mathrm{d}^{3} k}{(2 \pi)^{3}} \sum_{l \geq 2} \frac{(-1)^{l-1}}{l}\left(\frac{\hat{m}_{D}^{2}}{k^{2}}\right)^{l} \\
& =-\frac{T}{2} \int \frac{\mathrm{d}^{3} k}{(2 \pi)^{3}}\left[\log \left(1+\frac{\hat{m}_{D}^{2}}{k^{2}}\right)-\frac{\hat{m}_{D}^{2}}{k^{2}}\right]=\frac{\hat{m}_{D}^{3} T}{12 \pi},
\end{aligned}
$$

which is the order $-g^{3}$ contribution in eq. (8).

\subsubsection{Static resummation}

From the previous examples, we learned that (a) the occurrence of infrared divergences in the diagrammatic expansion calls for resummations and (b) 
it appears sufficient, in order to cure these divergences, to resum $\hat{m}_{D}^{2}$ in the static Matsubara modes alone.

Since $\hat{m}_{D}^{2}$ summarizes interaction effects, care should be taken to avoid overcounting in carrying out high order calculations within that scheme. One way to proceed systematically is to add and subtract a mass term for the static modes in the (imaginary-time) Lagrangian:

$$
\begin{aligned}
\mathcal{L} & \equiv \frac{1}{2}\left(\partial_{\tau} \phi\right)^{2}+\frac{1}{2}(\nabla \phi)^{2}+\frac{\lambda}{4 !} \phi^{4} \\
& =\frac{1}{2}\left(\partial_{\tau} \phi\right)^{2}+\frac{1}{2}(\nabla \phi)^{2}+\frac{1}{2} \hat{m}_{D}^{2} \phi_{0}^{2}+\frac{\lambda}{4 !} \phi^{4}+\Delta \mathcal{L},
\end{aligned}
$$

where $\Delta \mathcal{L} \equiv-\frac{1}{2} \hat{m}_{D}^{2} \phi_{0}^{2}$ and $\phi_{0}(\mathbf{x}) \equiv T \int_{0}^{\beta} d \tau \phi(\tau, \mathbf{x})$. This allows for a reorganization of the perturbation theory in which the tree-level amplitudes are generated by the first three terms in eq. (29) while $\Delta \mathcal{L}$ is treated formally as an additional interaction, of order $g^{2}$, to ensure that the HTL is not double counted in the static sector.

This procedure preserves a strict correspondence ${ }^{c}$ between diagrams (or well identified pieces of it) and powers of $g$. Thus, it is well suited for weak coupling expansions of static quantities, and it is indeed the scheme in which the higher-order calculations for the pressure and the screening mass alluded to before have been originally performed (for both scalar theory 35136137 and $\mathrm{QCD} \underline{4142143}$ ).

\subsubsection{Dimensional reduction}

The special role of the static mode $\phi_{0}$ may be handled in a more systematic fashion by constructing an effective theory in which the non-static modes $\phi_{\nu \neq 0}$ are 'integrated out' in perturbation theory. Specifically, one can write the thermal partition function as the following path-integral:

$$
Z=\mathcal{N} \int \mathcal{D}\left(\phi_{0}\right) \exp \left\{-S_{\text {eff }}\left[\phi_{0}\right]\right\}
$$

where

$$
\exp \left\{-S_{\text {eff }}\left[\phi_{0}\right]\right\}=\mathcal{N}^{\prime} \int \mathcal{D}\left(\phi_{\nu \neq 0}\right) \exp \left\{-\int_{0}^{\beta} \mathrm{d} \tau \int \mathrm{d}^{3} x \mathcal{L}_{E}(x)\right\},
$$

and $S_{\text {eff }}\left[\phi_{0}\right]$ is the effective action. Aside from the direct classical field contribution (i.e., the restriction of eq. (7) to the static mode $\phi_{0}$ ), this effective

${ }^{\mathrm{c}}$ This is true, strictly speaking, only if one uses dimensional regularization to control the ultraviolet divergences. 
action receives also contributions which, diagrammatically, correspond to connected diagrams whose external lines are associated to $\phi_{0}$, and the internal lines are the propagators of the non-static modes $\phi_{\nu \neq 0}$. Thus, a priori, $S_{\text {eff }}\left[\phi_{0}\right]$ contains operators of arbitrarily high order in $\phi_{0}$, which are also non-local. In practice, however, one wishes to expand $S_{\text {eff }}\left[\phi_{0}\right]$ in terms of local operators, i.e., operators with the schematic structure $a_{m, n} \nabla^{m} \phi_{0}^{n}$ with coefficients $a_{m, n}$ to be computed in perturbation theory.

It is then useful to introduce an intermediate scale $\Lambda(\Lambda \ll T)$ which separates $h a r d(k \gtrsim \Lambda)$ and soft $(k \lesssim \Lambda)$ momenta. All the non-static modes, as well as the static ones with $k \gtrsim \Lambda$ are hard (since $K^{2} \equiv \omega_{n}^{2}+k^{2} \gtrsim \Lambda^{2}$ for these modes), while the static $\left(\omega_{n}=0\right)$ modes with $k \lesssim \Lambda$ are soft. Thus, strictly speaking, in the construction of the effective theory along the lines indicated above, one has to integrate out also the static modes with $k \gtrsim \Lambda$. The benefits of this separation of scales are that $(a)$ the resulting effective action for the soft fields can be made local (since the initially nonlocal amplitudes can be expanded out in powers of $p / K$, where $p \ll \Lambda$ is a typical external momentum, and $K \gtrsim \Lambda$ is a hard momentum on an internal line), and (b) the effective theory is now used exclusively at soft momenta. This strategy, which consists of integrating out the nonstatic modes in perturbation theory in order to obtain an effective threedimensional theory for the soft static modes, is generally referred to as "dimensional reduction"

As an illustration let us consider a massless scalar theory with quartic interactions; that is, eq. (77) with $m=0$. The effective action for the soft fields takes the form:

$$
S\left[\phi_{0}\right]=\beta \mathcal{F}(\Lambda)+\int \mathrm{d}^{3} x\left\{\frac{1}{2}\left(\boldsymbol{\nabla} \phi_{0}\right)^{2}+\frac{1}{2} M^{2}(\Lambda) \phi_{0}^{2}+g_{3}^{2}(\Lambda) \phi_{0}^{4}+\cdots\right\},
$$

where $\mathcal{F}(\Lambda)$ is the contribution of the hard modes to the free-energy, and the dots stand for higher-order, local operators. We have changed the normalization of the field $\left(\phi_{0} \rightarrow \sqrt{T} \phi_{0}\right)$ so as to absorb the factor $\beta$ in front of the effective action. The parameters of the effective Lagrangian in eq. (32), like $M^{2}(\Lambda), g_{3}^{2}(\Lambda)$, etc., are computed in perturbation theory (they involve diagrams in which all the internal lines are hard), and depend on the separation scale $\Lambda$, the original coupling $g$, and the temperature $T$ (note that the effective theory depends on the temperature only through these parameters). To lowest order in $g, g_{3}^{2} \approx g^{2} T$, and $M \sim g T$, as we shall see shortly.

Note that the scale $\Lambda$ acts as an explicit ultraviolet (UV) cutoff for the loop integrals in the effective theory. Since it is arbitrary, the dependence on 
$\Lambda$ of soft loop contributions must cancel against the dependence on $\Lambda$ of the parameters in the effective action. Let us verify this cancellation explicitly in the case of the thermal mass $M$ of the scalar field, and to lowest order in perturbation theory. To this order, the mass parameter $M^{2}(\Lambda)$ in the effective action is obtained by integrating over hard momenta within the one-loop diagram in fig. 33 (cf. eq. (15)). This gives

$$
\begin{aligned}
M^{2}(\Lambda) & =12 g^{2} T \sum_{n} \int \frac{\mathrm{d}^{3} k}{(2 \pi)^{3}} \frac{\left(1-\delta_{n 0}\right)+\theta(k-\Lambda) \delta_{n 0}}{\omega_{n}^{2}+k^{2}} \\
& =12 g^{2} \int \frac{\mathrm{d}^{3} k}{(2 \pi)^{3}}\left\{\frac{n(k)}{k}+\frac{1}{2 k}-\theta(\Lambda-k) \frac{T}{k^{2}}\right\} .
\end{aligned}
$$

The first two terms within the last integral are the same as in eq. (15) with $m=0$. The first of them, involving the thermal distribution, gives the HTL contribution $\hat{m}_{D}^{2}=g^{2} T^{2}$, while the second one, involving $1 / 2 k$, is removed by the vacuum renormalization. The third term, involving the $\theta$-function, is easily evaluated. One finally gets:

$$
M^{2}(\Lambda)=\hat{m}_{D}^{2}-\frac{6 g^{2}}{\pi^{2}} \Lambda T \equiv g^{2} T^{2}\left(1-\frac{6}{\pi^{2}} \frac{\Lambda}{T}\right) .
$$

The $\Lambda$-dependent term above is subleading, by a factor $\Lambda / T \ll 1$.

The one-loop correction to the thermal mass within the effective theory is given by the same tadpole diagram, fig. 3 evaluated with the massive propagator $1 /\left(k^{2}+M^{2}(\Lambda)\right)$, coupling constant $g_{3}^{2} \approx g^{2} T$, and ultraviolet cut-off $\Lambda$. We obtain

$$
\begin{aligned}
\delta M^{2}(\Lambda) & =12 g^{2} T \int \frac{\mathrm{d}^{3} k}{(2 \pi)^{3}} \Theta(\Lambda-k) \frac{1}{k^{2}+M^{2}(\Lambda)} \\
& =\frac{6 g^{2}}{\pi^{2}} T \Lambda\left(1-\frac{\pi M}{2 \Lambda} \arctan \frac{M}{\Lambda}\right) \simeq \frac{6 g^{2}}{\pi^{2}} T \Lambda-\frac{3 g^{2}}{\pi} T \hat{m}_{D},
\end{aligned}
$$

where the terms neglected in the last step are of higher order in $\hat{m}_{D} / \Lambda$ or $\Lambda / T$.

As anticipated, the $\Lambda$-dependent terms cancel in the sum $M^{2} \equiv M^{2}(\Lambda)+$ $\delta M^{2}(\Lambda)$, which then provides the physical thermal mass within the present accuracy:

$$
M^{2}=M^{2}(\Lambda)+\delta M^{2}(\Lambda)=g^{2} T^{2}-\frac{3}{\pi} g^{3} T,
$$

which agrees again with eq. (23). 


\subsubsection{Screened perturbation theory}

Still another strategy has been used for taking into account the effects of the screening mass to all orders, rather than perturbatively. It consists of resumming the HTL $\hat{m}_{D}^{2}$ in all the Matsubara modes, static and nonstatic (as we did in subsection 2.2.1). It can be formulated via a simple generalization of eq. (29), viz.

$$
\mathcal{L}=\frac{1}{2} \partial_{\mu} \phi \partial^{\mu} \phi-\frac{1}{2} \hat{m}_{D}^{2} \phi^{2}-\frac{\lambda}{4 !} \phi^{4}+\Delta \mathcal{L}, \quad \Delta \mathcal{L} \equiv \frac{1}{2} \hat{m}_{D}^{2} \phi^{2},
$$

with $\Delta \mathcal{L}$ to be treated as a quantity of order $g^{2}$ in perturbative calculations. This scheme is commonly referred to as screened perturbation theory (SPT) 1820. To appreciate its virtues, and its limitations, it is again best to work out some examples.

We have obtained earlier the expression of the tadpole calculated with the dressed propagator (22) (cf. eqs. (23) and (18)):

$$
\Pi_{\text {tadpole }} \simeq 12 g^{2} I_{T}\left(\hat{m}_{D}\right) .
$$

We have seen that $\Pi_{\text {tadpole }}\left(\hat{m}_{D}=g T\right)$, when expanded in powers of $g$, reproduce the correct perturbative result to order $g^{3}$ (see eq. (23)). But, as $g$ grows, it deviates rapidly from this perturbative result, as can be seen in Fig. 7 The poor convergence of the weak coupling expansion exhibited here is related to that of the high-temperature expansion in eq. (18) which, as we have seen, involves in fact non analytical terms. By including the screening mass in the tree-level Lagrangian, and not treating it a perturbative quantity of order $g$, SPT provides a smooth extrapolation to large values of $g$, which represents a definite improvement over perturbation theory.

However, in contrast to the static resummation scheme based on eq. (29), the calculations within SPT generally involve temperaturedependent ultraviolet divergences. Of course, these are compensated by the "counterterm" $\Delta \mathcal{L}$, but only in all-order calculations. At any finite-order, the compensation holds only to the perturbative accuracy ${ }^{\mathrm{d}}$ of the calculation. For instance, in order to obtain (38), one has to introduce the mass counterterm $\delta m^{2}=-(\lambda / 2) I_{0}\left(\hat{m}_{D}\right)$, which depends on $T$, via $\hat{m}_{D}$. Specifically (with dimensional regularization, for definiteness, and $\bar{\mu}^{2}=4 \pi \mathrm{e}^{-\gamma} \mu^{2}$ ):

$$
\delta m^{2}=-12 g^{2} \mu^{\epsilon} \int \frac{\mathrm{d}^{3-\epsilon} k}{(2 \pi)^{3-\epsilon}} \frac{1}{2 \varepsilon_{k}\left(\hat{m}_{D}\right)} \simeq \frac{3 g^{2} \hat{m}_{D}^{2}}{4 \pi^{2}}\left(\frac{2}{\epsilon}+\ln \frac{\bar{\mu}^{2}}{\hat{m}_{D}^{2}}+1\right),
$$

dBy the "perturbative accuracy" of an all-order result we understand the highest order in $g$ that is correctly reproduced by the weak coupling expansion of that result. 


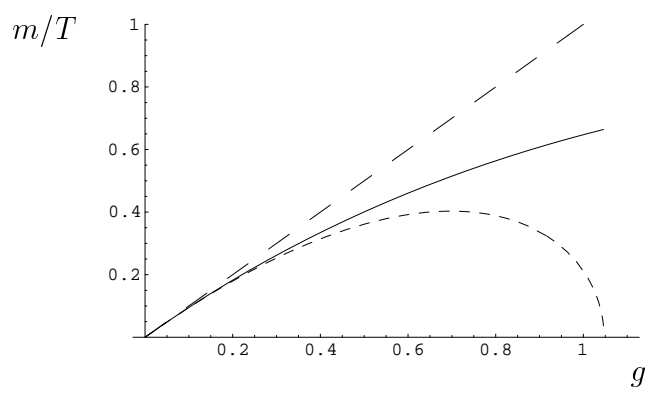

Fig. 7. The one-loop thermal mass in SPT, $m_{D}=\Pi_{\text {tadpole }}^{1 / 2}$, eq. 38, normalized to the temperature as a function of $g$ (full line). For comparison, we also present the perturbative estimates to order $g^{2}$ (long-dashed line) and $g^{3}$ (short-dashed line).

where $\varepsilon_{k}\left(\hat{m}_{D}\right)^{2}=k^{2}+\hat{m}_{D}^{2}$. This formula makes explicit the fact that the $T$-dependent divergence is of order $\mathcal{O}\left(g^{4}\right)$, and thus lies beyond the perturbative accuracy of this one-loop calculation, which is $\mathcal{O}\left(g^{3}\right)$.

As further illustration of the difficulties with SPT, let us compute the pressure to 2-loop order, and compare the result with its perturbative expansion to order $g^{3}$.

To one-loop order in the effective theory, the thermodynamic potential is given by eq. (13) with $m \rightarrow \hat{m}_{D}$. That is:

$$
\begin{aligned}
\frac{\Omega_{0}}{V} & =\frac{1}{2} T \sum_{n} \int \frac{\mathrm{d}^{3} k}{(2 \pi)^{3}} \ln \left(\omega_{n}^{2}+\mathbf{k}^{2}+\hat{m}_{D}^{2}\right) \\
& =\frac{1}{2} \int \frac{d^{3} k}{(2 \pi)^{3}} \varepsilon_{k}\left(\hat{m}_{D}\right)-P_{0}\left(\hat{m}_{D}\right)
\end{aligned}
$$

with $P_{0}(m)$ given by eq. (14):

$$
P_{0}\left(\hat{m}_{D}\right)=\frac{\pi^{2} T^{4}}{90}\left\{1-\frac{30}{8}\left(\frac{g}{\pi}\right)^{2}+\frac{15}{2}\left(\frac{g}{\pi}\right)^{3}+\mathcal{O}\left(g^{4} \ln g\right)\right\} .
$$

This overincludes the order $-g^{2}$ effect by a factor of 2 (compare to eq. (8)), but includes correctly the term of order $g^{3}$ (the fact that the plasmon effect is included correctly is somewhat accidental: If we were to resum not just the leading-order thermal mass $\hat{m}_{D}^{2}$, but also the NLO correction to it, $\Delta m_{D}^{2}=-(3 / \pi) g^{3} T^{2}$, one would modify the order $-g^{3}$ content of $\left.\Omega_{0}\right)$. The first piece in eq. (40), i.e., the sum of the "zero-point energies", is also UV divergent, and the divergent terms depends on $T$ since they depend on $\hat{m}_{D}$. To eliminate these divergences, we may introduce a temperature-dependent 
counterterm:

$$
\frac{\delta \Omega_{0}}{V}=-\frac{1}{2} \int \frac{d^{3} k}{(2 \pi)^{3}} \varepsilon_{k}\left(\hat{m}_{D}\right)=-\frac{1}{2} \int \frac{d^{4} k}{(2 \pi)^{4}} \ln \left(k_{0}^{2}+\mathbf{k}^{2}+\hat{m}_{D}^{2}\right),
$$

whose dependence upon $T$ starts already at order $g^{2}$. (This dependence can be pushed to order $g^{4}$, though, by using dimensional regularization.) This lowest order calculation reveals another unsatisfactory feature of SPT: the tree-level pressure calculated with the Lagrangian (37) fails to reproduce the lowest-order effect of the interactions.

Of course these drawbacks are partially corrected when we move to the next order, that is to two-loop order, which we do now. At this level, there are three contributions: the two-loop diagram in Fig. [4 and two one-loop diagrams involving mass counterterms, namely $\Delta \mathcal{L}$ in the Lagrangian (37), whose role is to correct for overcounting, and the ultraviolet counterterm $\delta m^{2}$, that has been introduced before, in eq. (39). Altogether:

$$
\frac{\Omega_{2}}{V}=\frac{\lambda}{8}\left[I\left(\hat{m}_{D}\right)\right]^{2}+\frac{1}{2}\left(\delta m^{2}-\hat{m}_{D}^{2}\right) I\left(\hat{m}_{D}\right) .
$$

It is convenient to combine the last counterterm with the tree-level expression $\Omega_{0}$ (written as in the first line of eq. (40)):

$$
\begin{aligned}
& \frac{\Omega_{0}}{V}-\frac{\hat{m}_{D}^{2}}{2} I\left(\hat{m}_{D}\right) \\
& =\frac{1}{2} T \sum_{n} \int \frac{\mathrm{d}^{3} k}{(2 \pi)^{3}}\left\{\ln \left(\omega_{n}^{2}+\mathbf{k}^{2}+\hat{m}_{D}^{2}\right)-\frac{\hat{m}_{D}^{2}}{\omega_{n}^{2}+\mathbf{k}^{2}+\hat{m}_{D}^{2}}\right\} .
\end{aligned}
$$

Clearly, the main effect of $\Delta \mathcal{L}$ is to cancel out the whole contribution of order $g^{2}$ that was originally present in the tree-level pressure. This includes the "anomalous" order $-g^{2}$ effect within $P_{0}\left(\hat{m}_{D}\right)$, and the divergent contribution of order $g^{2}$ that was eliminated by the counterterm (42) at one-loop order. The terms in eq. (43) can be simplified as follows:

$$
\frac{\lambda}{8}\left[I\left(\hat{m}_{D}\right)\right]^{2}+\frac{\delta m^{2}}{2} I\left(\hat{m}_{D}\right)=\frac{\lambda}{8}\left[I_{T}\left(\hat{m}_{D}\right)\right]^{2}-\frac{\lambda}{8}\left[I_{0}\left(\hat{m}_{D}\right)\right]^{2},
$$

(cf. eq. (19)). The last term is divergent and depends on the temperature, via $\hat{m}_{D}$, but this dependence counts only to order $g^{4}$, or higher. The order$g^{2}$ is obtained by replacing $I_{T}\left(\hat{m}_{D}\right)$ by $I_{T}(0)$ in the first term in eq. (45). The order $g^{3}$ receives contributions both from eq. (44) (as the lowest order correction beyond the ideal gas result), and from eq. (45) (as the nextto-leading order contribution). In both cases, the integration involves soft 
momenta only. Thus, we can restrict ourselves to the static mode $\omega_{n}=0$ in eq. (44), and get:

$$
\frac{1}{2} T \int \frac{\mathrm{d}^{3} k}{(2 \pi)^{3}}\left\{\ln \left(1+\frac{\hat{m}_{D}^{2}}{k^{2}}\right)-\frac{\hat{m}_{D}^{2}}{k^{2}+\hat{m}_{D}^{2}}\right\},
$$

while in eq. (45) we may proceed as follows:

$$
\begin{aligned}
\frac{\lambda}{8}\left(I_{T}\left(\hat{m}_{D}\right)\right)^{2}-\frac{\lambda}{8}\left(I_{T}(0)\right)^{2} & \simeq \frac{\lambda}{4} I_{T}(0)\left(I_{T}\left(\hat{m}_{D}\right)-I_{T}(0)\right) \\
& \simeq \hat{m}_{D}^{2} \frac{1}{2} T \int \frac{d^{3} k}{(2 \pi)^{3}}\left(\frac{1}{k^{2}+\hat{m}_{D}^{2}}-\frac{1}{k^{2}}\right)
\end{aligned}
$$

where we have also used eq. (26). Clearly, after adding the two contributions in eqs. (46) and (47), we are left with the standard expression for $P_{3}$, eq. (28).

Now, all the remaining ultraviolet divergences in eqs. (44) and (45) are to be cancelled by appropriate counterterms. One could be satisfied by the fact that the $T$-dependence of these counterterms starts at order $g^{4}$, which is beyond the perturbative accuracy of the present 2-loop calculation (namely, $\mathcal{O}\left(g^{3}\right)$ ). But leaving temperature dependence in UV counterterms is somewhat unphysical and would cause problems in nonperturbative calculations. Moreover, ambiguities arise from the choice of the subtraction scheme: One could e.g. either choose to subtract all the $T$-independent terms of the thermal pressure before identifying $\hat{m}_{D}$ with a thermal mass, or one may only subtract pole terms minimally on the grounds that also the terms not explicitly dependent on $T$ become so after making $\hat{m}_{D}$ temperature-dependent.

Some of these drawbacks may actually be absorbed by the flexibility in the choice of the mass parameter $\hat{m}_{D}$ in (37). Obviously, the results of SPT would be independent of this mass if they were obtained in an all order calculation. But for a finite-order calculation, the choice matters. One may try to optimize perturbation theory by a principle of minimal sensitivity resulting in a self-consistent gap equation 1820 . This has been shown to reduce greatly (though not completely) the dependence on the subtraction procedure ${ }^{51}$ (see also below, Fig. 9). Indeed, in Ref. 20 the calculation of the pressure of scalar $\phi^{4}$ theory has been extended to 2-loop order for the screening mass and 3 -loop order for the pressure (meaning a perturbative accuracy of order $g^{5}$ in both cases), with results showing an excellent apparent convergence: i.e., the difference between the 2-loop and 3 -loop results for the pressure remains rather small for all $g \lesssim 1$. 


\subsection{Self-consistent resummation}

\subsubsection{Skeleton expansion for thermodynamical potential}

A systematic way to take into account in the calculation of the thermodynamics, screening effects, or more generally propagator renormalisations, is to use the representation 52225354 of the thermodynamic potential $\Omega=-P V$ in terms of the full propagator $D$ :

$$
\beta \Omega[D]=-\log Z=\frac{1}{2} \operatorname{Tr} \log D^{-1}-\frac{1}{2} \operatorname{Tr} \Pi D+\Phi[D],
$$

where $\operatorname{Tr}$ denotes the trace in configuration space, $\beta=1 / T$, and $\Phi[D]$ is the sum of the two-particle irreducible (2PI) "skeleton" diagrams with no external legs:

$$
-\Phi[D]=1 / 12 \bigcirc+1 / 8 \bigcirc+1 / 48 \bigcirc+\ldots
$$

(where in the $\phi^{4}$ example the first diagram is absent of course). The full propagator $D$ is expressed in terms of the proper self-energy $\Pi$ by Dyson's equation ( $D_{0}$ is the free propagator):

$$
D=\frac{1}{D_{0}^{-1}+\Pi},
$$

and $\Pi$ itself, which is the sum of the one-particle irreducible (1PI) diagrams with two external lines, is obtained from $\Phi[D]$ by:

$$
\delta \Phi[D] / \delta D=\frac{1}{2} \Pi .
$$

By using this relation, one can check that $\Omega[D]$ is stationary under variations of $D$ (at fixed $D_{0}$ ) around the physical propagator:

$$
\delta \Omega[D] / \delta D=0 \quad \text { for } \quad D=\left(D_{0}^{-1}+\Pi\right)^{-1} .
$$

We shall usually refer to eq. (50) in which $\Pi$ is given in terms of $D$ by eq. (51) as a 'gap equation'.

An explicit expression for $\Omega$ is obtained by performing the summations over the Matsubara frequencies in eq. (48), using standard contour integration techniques. One obtains:

$$
\Omega / V=\int \frac{d^{4} k}{(2 \pi)^{4}} n(\omega)\left(\operatorname{Im} \log \left(-\omega^{2}+k^{2}+\Pi\right)-\operatorname{Im} \Pi D\right)+T \Phi[D] / V
$$


where $n(\omega)=1 /\left(\mathrm{e}^{\beta \omega}-1\right)$, and the imaginary parts are defined with retarded prescription. For instance:

$$
\operatorname{Im} D(\omega, k) \equiv \operatorname{Im} D(\omega+i \epsilon, k)=\frac{\rho(\omega, k)}{2},
$$

where $\rho(\omega, k)$ is the spectral function, which enters the following representation of the analytic propagator $D(\omega, k)$, valid for frequencies $\omega$ off the real axis:

$$
D(\omega, k)=\int_{-\infty}^{\infty} \frac{d k_{0}}{2 \pi} \frac{\rho\left(k_{0}, k\right)}{k_{0}-\omega} .
$$

The stationarity condition (52) can be used as a variational principle to deduce non-perturbative approximations for the physical propagator. A convenient approximation scheme of this type is the self-consistent (or " $\Phi$ derivable") approximation 22 obtained by selecting a class of skeletons in $\Phi[D]$ and calculating $\Pi$ from eq. (51). We shall see also that the stationarity property (52) brings simplifications in the calculation of the entropy.

\subsubsection{A simple model}

As a simple illustration of the general formalism of the previous subsection, we consider here the 2-loop approximation to $\Phi$, given by the first skeleton in the r.h.s. of eq. (49). ${ }^{\text {e }}$ The corresponding skeleton for $\Pi$ is the dressed tadpole (see Fig. B), and is local. Thus, the self-consistent self-energy is simply a mass, and we shall write $\Pi \equiv m^{2}$. (The vacuum mass is set to zero.) The gap equation (51) reads then:

$$
m^{2}=\frac{\lambda}{2} I(m)
$$

In contrast to the previous renormalization of eq. (38) (recall eq. (39)), it is possible to renormalize eq. (56) without introducing a thermal counterterm. This is done through coupling constant renormalization. To proceed, we first rewrite eq. (56) as (with the same notations as in eq. (391)):

$$
\mu^{\epsilon} m^{2}=-\lambda_{0} \frac{m^{2}}{32 \pi^{2}}\left(\frac{2}{\epsilon}+\log \frac{\bar{\mu}^{2}}{m^{2}}+1\right)+\lambda_{0} \int \frac{d^{3} k}{(2 \pi)^{3}} \frac{n\left(\varepsilon_{k}\right)}{2 \varepsilon_{k}}+\mathrm{O}(\epsilon),
$$

eThe self-consistent solution to 3-loop order has been worked out using mass expansions in Ref. 55 with satisfactory numerical results. However, the negative conclusion therein concerning renormalizability of $\Phi$-derivable approximations beyond two-loop order has since been refuted by Refs. 5657 . 
and relate $\lambda_{0}$ to the renormalized coupling $\lambda$ by:

$$
\frac{1}{\lambda}=\frac{\mu^{\epsilon}}{\lambda_{0}}+\frac{1}{16 \pi^{2} \epsilon}
$$

Then, the equation takes the following, manifestly finite, form (for $\epsilon \rightarrow 0$ ):

$$
m^{2}=\frac{\lambda}{2} \int \frac{\mathrm{d}^{3} k}{(2 \pi)^{3}} \frac{n\left(\varepsilon_{k}\right)}{\varepsilon_{k}}+\frac{\lambda m^{2}}{32 \pi^{2}}\left(\log \frac{m^{2}}{\bar{\mu}^{2}}-1\right) .
$$

One should stress a subtle point in the procedure: expressing the bare coupling $\lambda_{0}$ in terms of the renormalized one $\lambda$ according to eq. (58) is not sufficient to render finite the r.h.s. of eq. (57) for arbitrary values of $\mathrm{m}^{2}$, but only for these values which satisfies the finite equation (59).

Note that the 1-loop correction to the 4-point function that is considered here is iterated only in one channel among the three possible ones. Accordingly, the $\beta$-function for the renormalized coupling constant (58):

$$
\frac{\mathrm{d} \lambda}{\mathrm{d} \log \bar{\mu}}=\frac{\lambda^{2}}{16 \pi^{2}},
$$

(this follows by noticing that $\lambda_{0}$ is independent of $\bar{\mu}$ in eq. (58)) is only one third of the lowest-order perturbative $\beta$-function for this scalar field theory (see eq. (9)). This is no actual fault since the running of the coupling affects the thermodynamic potential only at order $\lambda^{2}$ which is beyond the perturbative accuracy of the 2-loop $\Phi$-derivable approximation. (In order to see the correct one-loop $\beta$-function, the approximation for $\Phi$ would have to be pushed to 3-loop order.) By using eq. (60), one can check that the solution $m^{2}$ of eq. (59) is independent of $\mu$.

By using the previous formulae, one can easily compute the pressure 58 .

$$
P=-T \int \frac{\mathrm{d}^{3} k}{(2 \pi)^{3}} \log \left(1-\mathrm{e}^{-\beta \varepsilon_{k}}\right)+\frac{m^{2}}{2} \int \frac{d^{3} k}{(2 \pi)^{3}} \frac{n\left(\varepsilon_{k}\right)}{2 \varepsilon_{k}}+\frac{m^{4}}{128 \pi^{2}},
$$

which differs from the pressure $P_{0}(m)$ of an ideal gas of massive particles, eq. (14), by the last two terms in the r.h.s.

By construction, the 2-loop $\Phi$-derivable approximation is perturbatively correct to order $g^{3}$, and, indeed, it can be easily verified that the weak coupling expansions of eqs. (59) and (61) include the correct perturbative effects of order $g^{2}$ and $g^{3}$ (together with incomplete contributions of higher orders). But the complete, self-consistent results, as obtained by numerical evaluation in eqs. (59) and (61), show a much better behaviour than the respective perturbative expansions to order $g^{3}$, in the sense of being monotonic with $g$ (unlike the perturbative approximants of order $g^{3}$ ), 


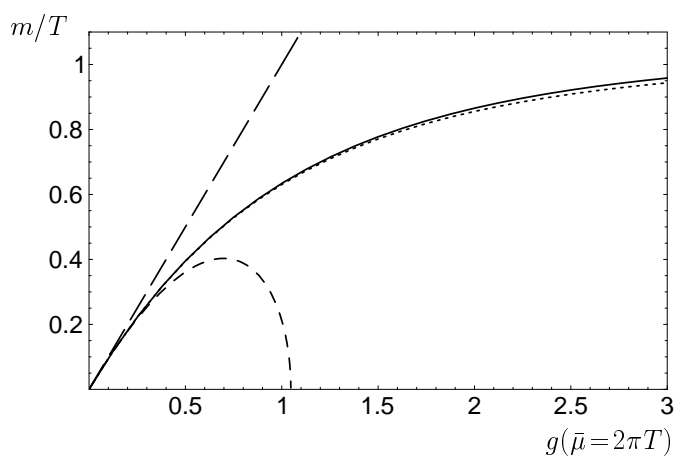

Fig. 8. Numerical solution of the mass gap equation [59] with $\bar{\mu}=2 \pi T$ in comparison with the perturbative result to order $g^{2}$ and $g^{3}$ for $m$ (long and medium dashed lines, resp.). The short-dashed line just below the full line corresponds to the quadratic gap equation 65 following from the requirement of only soft self-consistency.

and showing saturation at very large $g \gtrsim 2$ (in contrast to the order- $g^{2}$ estimates). This behaviour is illustrated in Figs. 8 and 9 for the self-consistent mass (59) and pressure (61), respectively.

Also shown in Fig. [1 is the result of SPT at two-loop order when the mass parameter is determined by a principle of minimal sensitivity. It turns out that for a minimal subtraction of the additional UV divergences of SPT and identification of the corresponding regularization mass scale with that used in the $(\overline{\mathrm{MS}})$ renormalization of the coupling constant, the result of SPT coincides with that of the 2-loop $\Phi$-derivable approximation. However, if the additional UV divergences of SPT are subtracted differently, this coincidence no longer occurs. As an example, in Fig. 9 the grey full line displays the SPT result with a "maximal" subtraction of the additional UV divergences where $P(T)$ is made finite by subtracting $P(0)$ when treating $\hat{m}_{D}$ as temperature-independent (since it is just a parameter in the Lagrangian), and only afterwards turning it temperature-dependent by fixing $\hat{m}_{D}$ by a variational principle. Although (for $N=1$ scalar theory) one cannot decide how the exact result would look like, the result appears to be less satisfactory in that it exceeds the free pressure for $g>1.5$. The prescription that has been used in the application of SPT to QCD is in fact that of a minimal subtraction of the additional UV divergences of SPT 215960 .

We have previously argued that the expected accuracy of perturbation theory is a priori different in the soft and the hard sectors. Within the present solvable model, it is possible to verify this argument explicitly. To 


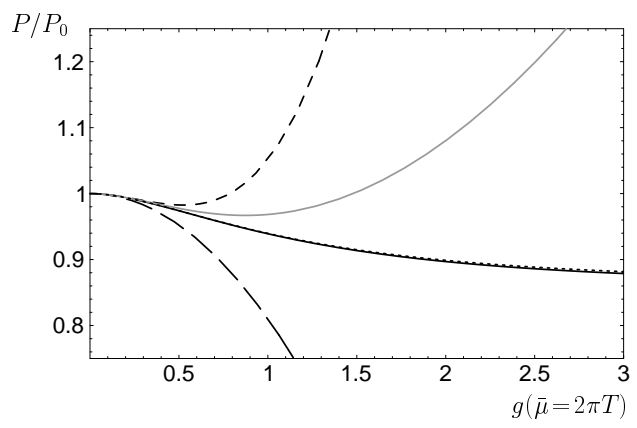

Fig. 9. The 2-loop $\Phi$-derivable pressure 61 in comparison with the perturbative results to order $g^{2}$ and $g^{3}$ (long and medium dashed lines, resp.). The short-dashed line just above the full line corresponds to the pressure evaluated perturbatively for hard modes and self-consistently only for the soft modes, eqs. 62 and 66 with 65. SPT at 2-loop order turns out to coincide with the 2-loop $\Phi$-derivable given by the full line, when the former is subtracted minimally, but gives different results (grey full line) when subtracted "maximally" (see text).

this aim, we shall reformulate the self-consistent calculation of the thermal mass and the pressure in an approximate way, exploiting the distinction between the hard and soft sectors. That is, we shall build on the strategy of effective theories to obtain perturbatively, through a calculation in the hard sector, the coefficients of an effective Lagrangian for the soft sector. We shall then look for an "exact" solution in the soft sector, which amounts here to use the two-loop self-consistent approximation. As we shall see, this strategy, which mimics that used in calculations based on dimensional reduction, turns out to be remarkably accurate. It also provides a clearer physical justification for approximations that have been motivated previously by other considerations.

Thus, we shall approximate the fully self-consistent 2-loop result for the pressure, eq. (61), by $P=P_{(2)}+P_{\mathrm{s}}$, where $P_{(2)}$ is the perturbative result to order $g^{2}$, which is due to the hard modes alone:

$$
P_{(2)}=\frac{\pi^{2}}{90} T^{4}\left[1-\frac{15}{8}\left(\frac{g}{\pi}\right)^{2}\right] .
$$

and $P_{\mathrm{s}}$ is obtained via a $2-$ loop self-consistent calculation within the effective three-dimensional theory with Lagrangian (32). To the order of interest, one can set $M^{2} \approx \hat{m}_{D}^{2}$ and $g_{E}^{2} \approx g^{2} T$ in eq. (32), and neglect all the other interaction vertices. 
The corresponding free-energy functional reads then:

$$
\begin{aligned}
\frac{\beta \Omega_{\mathrm{s}}\left[\Pi_{\mathrm{s}}\right]}{V}= & \frac{1}{2} \int_{k}\left\{\ln \left(k^{2}+\hat{m}_{D}^{2}+\Pi_{\mathrm{s}}\right)-\frac{\Pi_{\mathrm{s}}}{k^{2}+\hat{m}_{D}^{2}+\Pi_{\mathrm{s}}}\right\} \\
& +\frac{\lambda}{8}\left(\int_{k} \frac{1}{k^{2}+\hat{m}_{D}^{2}+\Pi_{\mathrm{s}}}\right)^{2},
\end{aligned}
$$

where $\int_{k}$ denotes the momentum integral in $3-d$ dimensions. Note that, as compared to Sect. 2.2.4 here we find it more convenient to use dimensional regularization, with the scale $\mu$ chosen as the separation scale $\Lambda$ between soft and hard momenta. This has the advantage that all the power-like divergences in integrals like those in eq. (63) are now set to zero, so, conversely, the parameters of the effective Lagrangian are independent of $\Lambda$ to the accuracy of interest 37 .

In eq. (63), $\Pi_{\mathrm{s}}$ is the scalar self-energy within the $3-d$ effective theory, and is determined from a gap equation which follows by demanding $\Omega_{\mathrm{S}}\left[\Pi_{\mathrm{s}}\right]$ to be stationary with respect to $\Pi_{\mathrm{s}}$ :

$$
\Pi_{\mathrm{s}}=\frac{\lambda}{2} T \int_{k} \frac{1}{k^{2}+\hat{m}_{D}^{2}+\Pi_{\mathrm{s}}},
$$

or, equivalently, with $m^{2} \equiv \hat{m}_{D}^{2}+\Pi_{\mathrm{s}}$,

$$
m_{\mathrm{s}}^{2}=\hat{m}_{D}^{2}-\frac{3}{\pi} g^{2} T m_{\mathrm{s}} .
$$

This is a second-order algebraic equation for $m_{\mathrm{s}}$, whose r.h.s. is recognized as the expansion of the integral in the general gap equation in four dimensions, eq. (56), up to terms linear in $m / T$. Note that, to this accuracy, there is no ultraviolet divergence at all, so the coupling constant $\lambda$ (or $g^{2}$ ) in the equations above is the renormalized one.

The free energy (63) can be easily evaluated in terms of the solution $m_{\mathrm{s}}$ to the above gap equation. One obtains $\left(P_{\mathrm{s}}=-\Omega_{\mathrm{s}} / V\right)$ :

$$
P_{\mathrm{s}}=\frac{T m_{\mathrm{s}}^{3}}{12 \pi}-\frac{T m_{\mathrm{s}}}{8 \pi}\left(m_{\mathrm{s}}^{2}-\hat{m}_{D}^{2}\right)-\frac{3}{16}\left(\frac{g}{\pi}\right)^{2} T^{2} m_{\mathrm{s}}^{2} .
$$

Note that this contribution starts at order $g^{3}$, but includes terms of all higher orders, via $m_{\mathrm{s}}$. The sum $P=P_{(2)}+P_{\mathrm{s}}$ of eqs. (62) and (66) should be compared to the fully self-consistent result in eq. (61). Clearly, these two approximations coincide up to order $g^{3}$, but deviate in higher orders, with the deviations restricted, however, to contributions due to the hard modes. In Figs. 8 and 9 we also compare the numerical solutions to the full gap equation (59) and its "soft" approximation (65), and also the corresponding 
predictions for the pressure. We see that these predictions are extremely close to each other, for both $m$ and $P$, which confirms that the higher order effects associated with the hard modes are small indeed.

\subsubsection{The entropy}

We have mentioned already that the stationarity property (52) allows one to simplify the calculation of the entropy:

$$
\mathcal{S}=-\partial(\Omega / V) / \partial T
$$

or, more generally, of any first partial derivative of the pressure. Indeed, the thermodynamic potential (53) depends on the temperature through the statistical factors $n(\omega)$ and the propagator, or equivalently its spectral function $\rho$. Because of eq. (52) the temperature derivative of the spectral density cancels out and one obtains 6126 .

$$
\begin{aligned}
\mathcal{S}= & -\int \frac{d^{4} k}{(2 \pi)^{4}} \frac{\partial n(\omega)}{\partial T} \operatorname{Im} \log D^{-1}(\omega, k) \\
& +\int \frac{d^{4} k}{(2 \pi)^{4}} \frac{\partial n(\omega)}{\partial T} \operatorname{Im} \Pi(\omega, k) \operatorname{Re} D(\omega, k)+\mathcal{S}^{\prime}
\end{aligned}
$$

with

$$
\mathcal{S}^{\prime} \equiv-\left.\frac{\partial(T \Phi)}{\partial T}\right|_{D}+\int \frac{d^{4} k}{(2 \pi)^{4}} \frac{\partial n(\omega)}{\partial T} \operatorname{Re} \Pi \operatorname{Im} D
$$

Loosely speaking, the first two terms in eq. (68), which have a one-loop structure, represent the entropy of 'independent quasiparticles', while $\mathcal{S}^{\prime}$ accounts for a residual interaction among these quasiparticles 26 (see also the discussion below). For 2-loop ${ }^{\mathrm{f}}$ skeletons we have the following additional simplification 612625 .

$$
\mathcal{S}^{\prime}=0,
$$

a property which holds independently of the self-consistency condition (52).

The formula (68), together with $\mathcal{S}^{\prime}=0$, makes the entropy a privileged quantity to study the thermodynamics of ultrarelativistic plasmas. Let us discuss some of the attractive features.

${ }^{\mathrm{f}}$ For the scalar field theory with $\phi^{4}$ self-interactions, the property 3-loop skeleton in eq. 49. 
First, we note that, for a finite function $\Pi(\omega, k)$, the integrals in eq. 688 with $\mathcal{S}^{\prime}=0$ are manifestly ultraviolet finite, since $\partial n / \partial T$ vanishes exponentially for both $\omega \rightarrow \pm \infty$. This reduces the problem of the UV renormalization of the 2-loop $\Phi$-derivable approximation for the entropy to that of the renormalization of the gap equation for the 1-loop self-energy. In general, this is still a difficult task (because this is a non-perturbative approximation), but as we have seen in the previous subsection, progress can be made at least in specific cases. In particular, it has been recently

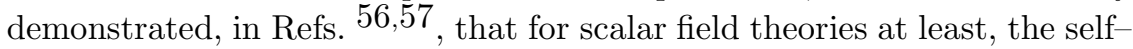
consistent approximations are renormalizable to all orders, i.e., they can be made finite by adding temperature-independent (vacuum) counterterms to the Lagrangian, which are themselves determined in a self-consistent way.

Furthermore, as already mentioned, the entropy has a more direct quasiparticle interpretation than the pressure. To see this more explicitly, use the following identity:

$$
\operatorname{Im} \log D^{-1}(\omega, k)=\arctan \left(\frac{\operatorname{Im} \Pi}{\operatorname{Re} D^{-1}}\right)-\pi \epsilon(\omega) \theta\left(-\operatorname{Re} D^{-1}\right),
$$

to rewrite $\mathcal{S}$ as $\mathcal{S}=\mathcal{S}_{\text {pole }}+\mathcal{S}_{\text {damp }}$, with

$$
\begin{aligned}
\mathcal{S}_{\text {pole }} & =\int \frac{d^{4} k}{(2 \pi)^{4}} \frac{\partial n(\omega)}{\partial T} \pi \epsilon(\omega) \theta\left(-\operatorname{Re} D^{-1}(\omega, k)\right) \\
& =\int \frac{d^{3} k}{(2 \pi)^{3}}\left\{\left(1+n_{k}\right) \log \left(1+n_{k}\right)-n_{k} \log n_{k}\right\},
\end{aligned}
$$

where $n_{k} \equiv n\left(\varepsilon_{k}\right)$, with $\varepsilon_{k}$ solution of $\operatorname{Re} D^{-1}\left(\omega=\varepsilon_{k}, k\right)=0$. This is the on-shell energy of the dressed single-particle excitations in the system, or 'quasiparticles'. The quantity $\mathcal{S}_{\text {pole }}$ is the entropy of a system of 'noninteracting' quasiparticles, while the quantity

$$
\mathcal{S}_{\text {damp }}=\int \frac{d^{4} k}{(2 \pi)^{4}} \frac{\partial n(\omega)}{\partial T}\left[\operatorname{Im} \Pi \operatorname{Re} D-\arctan \left(\frac{\operatorname{Im} \Pi}{\operatorname{Re} D^{-1}}\right)\right],
$$

which vanishes when $\operatorname{Im} \Pi$ vanishes, is a contribution coming from the continuum part of the quasiparticle spectral weights. We see that for the entropy, unlike for the pressure, the main effects of the interactions come via the change in the spectrum of the excitations, and therefore can be taken into account by simply dressing the propagator.

The above formulae, which are generic (i.e., they hold for any field theory, including QCD), become even simpler when the 2-loop self-consistent approximation is applied to the scalar theory with $\phi^{4}$ self-interactions. Then, the self-energy reduces to a local mass term, as we have seen in the 
previous subsection, and therefore $\mathcal{S}=\mathcal{S}_{\text {pole }}$, with $\mathcal{S}_{\text {pole }}$ given by eq. (72) with $\varepsilon_{k}^{2}=k^{2}+m^{2}$ and $m$ determined by eq. (59). That is, in this approximation, the entropy of the interacting scalar gas is formally identical to the entropy of an ideal gas of massive bosons, with mass $m$. As emphasized after eq. 61), such a simple interpretation does not hold for the pressure.

\subsubsection{Approximately self-consistent calculations of the entropy}

In this subsection, the calculation of the self-consistent 2-loop entropy will be considered from a new perspective, which will suggest a different strategy for implementing the self-consistency in an approximate way. This strategy will be especially useful in view of subsequent applications to QCD, where a fully self-consistent calculation seems prohibitively difficult, and not even desirable (see Sect. 4.2).

It is first instructive to see how the perturbative effects of order $g^{2}$ and $g^{3}$ get built within the self-consistent 2-loop entropy, as given by (cf. eq. (72)):

$$
\begin{aligned}
\mathcal{S}(m) & =-\int \frac{d^{4} k}{(2 \pi)^{4}} \frac{\partial n(\omega)}{\partial T} \operatorname{Im} \log \left(k^{2}-\omega^{2}+m^{2}\right) \\
& =\frac{2 \pi^{2} T^{3}}{45}-\frac{m^{2} T}{12}+\frac{m^{3}}{12 \pi}+\mathcal{O}\left(m^{4} / T\right) .
\end{aligned}
$$

The first two terms in the expansion in the second line come from integrating over hard momenta $(k \sim T)$ within the loop integral in the first line, while the third term, non-analytic in $m^{2}$, comes from the soft loop $(k \sim m)$.

The expansion in eq. (74) must be considered simultaneously with the perturbative expansion of the solution $m$ to the gap equation (59). To order $g^{3}$, this is the same as the standard perturbative expansion, eq. (23): $m^{2} \simeq g^{2} T^{2}(1-3 g / \pi)$.

If one replaces $m$ in eq. (74) by the LO thermal mass $\hat{m}_{D}=g T$, then the resulting approximation for $\mathcal{S}$ reproduces the perturbative effect of order $g^{2}$, but underestimates the correction of order $g^{3}$ by a factor of 4 . But to order $g^{3}$, the thermal mass itself must be corrected, as discussed above. By replacing $m^{2} \rightarrow \hat{m}_{D}^{2}+\delta m_{D}^{2}$ in the second order term of eq. (74), the correction $\delta m_{D}^{2}$ to the thermal mass generates the $3 / 4$ of the plasmon effect missing. To conclude, the order $-g^{3}$ contribution to the entropy comes up in a subtle way: $1 / 4$ of it comes directly from the soft loop in eq. (74), with $m$ replaced by the LO screening mass $\hat{m}_{D}$, while the other $3 / 4$ comes from 


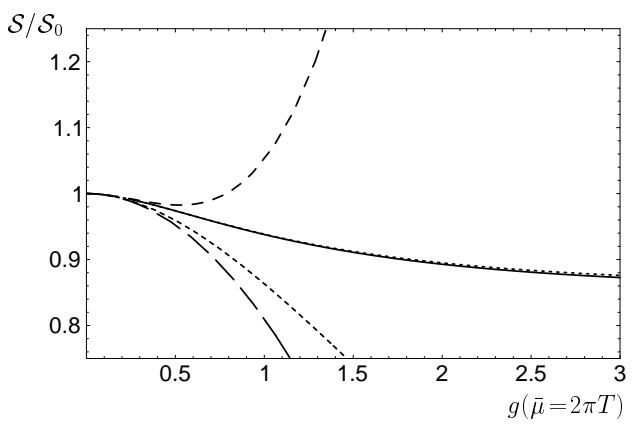

Fig. 10. Comparison of the fully self-consistent 2-loop entropy with the perturbative results to order $g^{2}$ and $g^{3}$ (long and medium dashed lines, resp.). The short-dashed line between the order $g^{2}$ result and the full line corresponds to the "HTL approximation" where the 2-loop entropy is evaluated with just the HTL mass; the short-dashed line almost on top of the full line corresponds to the "NLA approximation" using (76).

the hard loop, via the NLO correction to the dispersion relation of the hard particles.

Of course, within the present context, where the self-energy is independent of the external momentum, there is no distinction between the screening mass and the quasiparticle mass: both are solution to the same gap equation (59). But in QCD, these are distinct quantities already at lowest order, and this difference will play an important role in the construction of approximations in Sect. 4 Thus, although this may look at first somewhat artificial here, we shall introduce different notations for the two masses. We shall keep the notation $m_{D}$ for the screening (Debye) mass, and denote the hard quasiparticle mass as $m_{\infty}$ : the latter characterizes the excitation spectrum at large momenta, typically of the form $\varepsilon_{k}=\sqrt{k^{2}+m_{\infty}^{2}}$. Thus the mass $m^{2}$ in the second order term of eq. (74) should be read as $m_{\infty}^{2}$, while $m^{3}$ in the cubic term is $m_{D}^{3}$. That is, we rewrite eq. (74) as

$$
\mathcal{S}(m) \simeq \frac{2 \pi^{2} T^{3}}{45}-\frac{m_{\infty}^{2} T}{12}+\frac{m_{D}^{3}}{12 \pi}
$$

In perturbation theory, $m_{D}$ and $m_{\infty}$ coincide with each other up to order $g^{3}$, but differ in order $g^{4}$ and higher. ${ }^{9}$ Within the 2-loop $\Phi$-derivable approximation, they coincide if computed exactly, as already mentioned.

gNote that only the corrections to $m_{D}$ can be calculated by means of dimensional reduction. Dynamical masses in general require the resummation of the nonstatic hard thermal loops (for simple examples see Ref. 62 ). 
But if the self-consistency is imposed only in the soft sector, the corresponding approximations may a priori look different. We have seen earlier that self-consistency in the soft sector is enough to obtain an excellent approximation for the pressure, and we want to verify here the status of such an approximation for the entropy. In fact we shall examine the accuracy of two non-perturbative approximations to the entropy, that we shall later implement in the case of QCD. In both approximations, the integral in the first line of eq. (74) is evaluated exactly (i.e., numerically), but for values of $m$ which are only approximate solutions to the gap equation.

$i$ ) In the 'HTL approximation' we set $m=\hat{m}_{D}$. The ensuing approximation for $\mathcal{S}$ includes the correct LO effect of the interactions, of order $g^{2}$, but only $1 / 4$ of the plasmon effect. In Fig. 10, the resulting approximation is compared to the exact (i.e., fully self-consistent) result, and also to the weak coupling expansion.

ii ) In the 'next-to-leading approximation' (NLA), the mass $m_{\infty}$ of the hard particles is determined with NLO accuracy, so as to restore the correct plasmon effect in $\mathcal{S}$. That is,

$$
m_{\infty}^{2} \longrightarrow m_{\infty}^{2}-(3 / \pi) g^{2} T m_{D},
$$

where $m_{D}$ is the self-consistent mass, given by the solution to the gap equation (65). Of course, the mass $m_{\infty}$ thus obtained coincides with the Debye mass $m_{D}$, but this 'degeneracy' is specific to the present scalar model, as we have already emphasized.

The quality of the NLA based on eq. (76) can be appreciated from its comparison, in Fig. 10] with the "exact" (fully self-consistent) result, and also with the HTL approximation alluded to before. Although the HTL approximation is seen to deviate from the fully self-consistent result at large coupling, it remains smaller than the ideal-gas result despite the presence of a quarter of the plasmon effect, which, when treated purely perturbatively, would result in a nonmonotonic behaviour leading to an excess over the ideal-gas result at large coupling.

The $\Phi$-derivable 2-loop approximation in fact becomes "exact" in the large- $N$ limit, where $N$ scalars are interacting in an $\mathrm{O}(N)$-invariant manner according to $\left(\vec{\phi}^{2}\right)^{2}$. In this limit the exact result is $\bar{\mu}$-independent, while all approximations to it are renormalization scale dependent. However, as can be seen in Fig. 10 the agreement is almost perfect for NLA when $\bar{\mu}=2 \pi T$ and varies only slightly when varying this scale, which motivates us to adopt $\bar{\mu}=2 \pi T$ as optimal renormalization point. However, in what follows we shall always consider variations by a factor of 2 around this central value 
to have an estimate of the inherent uncertainty of our approximations.

\section{Quark-Gluon Plasma}

Before describing how to overcome the difficulties of perturbation theory in the thermodynamics of QCD along the lines sketched for the simple scalar models of the previous section, we shall briefly review the results of perturbation theory obtained so far, which, at least for vanishing chemical potential and high temperature, have been recently pushed to the limits of calculability.

\subsection{Perturbative results}

In QCD with $N_{f}$ flavors of quarks, the thermodynamic pressure at high temperature has been computed $\frac{4142}{14}$ in perturbation theory through order $g^{5}$ :

$$
\begin{aligned}
P=\frac{8 \pi^{2}}{45} T^{4}\{ & \left(1+\frac{21}{32} N_{f}\right)+\frac{-15}{4}\left(1+\frac{5}{12} N_{f}\right) \frac{\alpha_{s}}{\pi} \\
+ & 30\left[\left(1+\frac{N_{f}}{6}\right)\left(\frac{\alpha_{s}}{\pi}\right)\right]^{3 / 2} \\
+ & \left\{237.2+15.97 N_{f}-0.413 N_{f}^{2}+\frac{135}{2}\left(1+\frac{N_{f}}{6}\right) \ln \left[\frac{\alpha_{s}}{\pi}\left(1+\frac{N_{f}}{6}\right)\right]\right. \\
& \left.-\frac{165}{8}\left(1+\frac{5}{12} N_{f}\right)\left(1-\frac{2}{33} N_{f}\right) \ln \frac{\bar{\mu}}{2 \pi T}\right\}\left(\frac{\alpha_{s}}{\pi}\right)^{2} \\
+(1+ & \left.\frac{N_{f}}{6}\right)^{1 / 2}\left[-799.2-21.96 N_{f}-1.926 N_{f}^{2}\right. \\
+ & \left.\frac{495}{2}\left(1+\frac{N_{f}}{6}\right)\left(1-\frac{2}{33} N_{f}\right) \ln \frac{\bar{\mu}}{2 \pi T}\right]\left(\frac{\alpha_{s}}{\pi}\right)^{5 / 2} \\
+ & \left.\mathcal{O}\left(\alpha_{s}^{3} \ln \alpha_{s}\right)\right\},
\end{aligned}
$$

where $\alpha_{s}=g^{2}(\bar{\mu}) /(4 \pi)$. The coefficient of the $\alpha_{s}^{3} \ln \alpha_{s}$ term, the last in the pressure at high $T$ and vanishing chemical potential that is calculable in perturbation theory, has recently been determined as 63

$$
\begin{aligned}
\left.P\right|_{g^{6} \ln g}= & \frac{8 \pi^{2}}{45} T^{4}\left[1134.8+65.89 N_{f}+7.653 N_{f}^{2}\right. \\
& \left.-\frac{1485}{2}\left(1+\frac{N_{f}}{6}\right)\left(1-\frac{2}{33} N_{f}\right) \ln \frac{\bar{\mu}}{2 \pi T}\right]\left(\frac{\alpha_{s}}{\pi}\right)^{3} \ln \frac{1}{\alpha_{s}} .
\end{aligned}
$$


Here $\bar{\mu}$ is the renormalization scale parameter of the $\overline{\mathrm{MS}}$ scheme and $\alpha_{s}(\bar{\mu})$ is the running coupling whose beta function is known to four-loop order 64 , though we shall restrict ourselves to its two-loop version in the following, because lattice determinations of $T_{c} / \Lambda_{\overline{\mathrm{MS}}}$ usually employ only the latter.

Evaluating the perturbative expression to order $g^{2}, g^{3}, g^{4}$, and $g^{5}$ gives results which do not show any sign of convergence, as depicted in Fig. 11] but rather show increasing ambiguities due to the dependence on the renormalization point, signalling a complete loss of predictive power. (When the $g^{6} \ln g$-term of Eq. (78) is included with a suitably adjusted constant under the log, the latter can at least be chosen such that the lattice results are qualitatively reproduced 63 .)

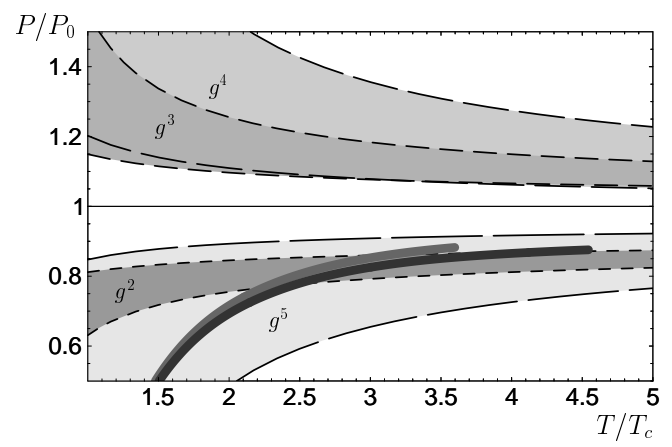

Fig. 11. The poor convergence of thermal perturbation theory in pure glue QCD. The various grey bands bounded by differently dashed lines show the perturbative results to order $g^{2}, g^{3}, g^{4}$, and $g^{5}$ with $\overline{\mathrm{MS}}$ renormalization point $\bar{\mu}$ varied between $\pi T$ and $4 \pi T$. The thick dark-grey line shows the lattice results from the Bielefeld group 65 , the lighter one behind that of a more recent lattice calculation using an RG-improved action from the CP-PACS collaboration 66

However, the poor convergence behaviour of perturbation theory is not very different from that we have observed above in the case of simple scalar $\phi^{4}$ theory. In the following we shall review the attempts to overcome this impasse by reorganizing perturbation theory on the one hand by a variant of SPT and on the other by an approach which is guided by $\Phi$-derivable approximations for entropy and quark number density. In both cases, the resulting expressions go beyond strict perturbation theory in that higherorder terms are kept unexpanded, and the hope is that strictly nonperturbative contributions that appear in the pressure of hot QCD at order $g^{6}$ 
make up only a small, negligible part of what can be captured already by an effective quasiparticle description as soon as the temperature is a few times the transition temperature.

When compared with the perturbative results, it is remarkable that the next-to-leading result to order $g^{2}$ fares rather well at temperatures $\gtrsim 2 T_{c}$, though the higher-order results prove that perturbation theory is inconclusive. On the other hand, simple quasiparticle models which describe the effective gluonic degrees of freedom by $2 N_{g}\left(N_{g}=N^{2}-1\right)$ scalar degrees of freedom with asymptotic masses taken from a HTL approximation can be used quite successfully to model the lattice data by fitting the running coupling 27282930 .

\subsection{Lattice results}

The thermodynamic potential has been studied on 4-dimensional lattices both for pure-glue QCD and QCD with a number of comparatively light (but still massive) quarks by a number of groups.

In the case of pure-glue QCD, the temperature dependence of the thermal pressure has been studied in great detail on lattices with varying temporal extent up to $8 \times 32^{3}$ for temperatures up to about $4.5 T_{c}$. The continuum extrapolation using the standard Wilson action of Ref. 65 is given in Fig. 11 by the darker-grey line with the width of the line roughly representing the estimated error. Similar studies have been performed using improved lattice actions 6766 and the results obtained in Ref. $\frac{66}{6 s i n g}$ a renormalization-group improved lattice action are included in Fig. 11 by the lighter-grey line. The latter are systematically higher by about $5 \ldots 2 \%$ for temperatures $2 \ldots 3.5 T_{c}$ compared to the results of Ref. 65 .

As can be seen in Fig. 11] the pressure drops sharply as the transition temperature is approached from above. Not shown in the figure is that the pressure in fact drops to virtually zero at and below the transition temperature. There the pressure is exponentially suppressed by the rather large masses of glueballs.

In the presence of (light) quarks, lattice calculations are much more difficult to perform and the continuum extrapolation is correspondingly more involved and uncertain. However, through the use of improved lattice action, considerable progress has been made recently $\underline{6869}$. When rescaled to the ideal-gas values, the results, as a function of $T / T_{c}$ with the respective different values of $T_{c}$, the pattern of the pressure of hot QCD with quarks is quite similar to that of pure-glue QCD, and there is moreover a rather 
weak dependence on the number of flavours if all of them are light.

\subsection{Dimensional reduction}

Dimensional reduction in hot QCD (at zero chemical potential) leads to an effective three-dimensional Lagrangian $\underline{454647}$

$$
\mathcal{L}_{E}=\frac{1}{2} \operatorname{tr} F_{i j}^{2}+\operatorname{tr}\left[D_{i}, A_{0}\right]^{2}+m_{E}^{2} \operatorname{tr} A_{0}^{2}+\frac{1}{2} \lambda_{E}\left(\operatorname{tr} A_{0}^{2}\right)^{2}+\ldots
$$

where the parameters are determined perturbatively by matching 43170 . In lowest order one has a dimensionful coupling $g_{E}^{2}=g^{2} T$ and 47

$$
m_{E}^{2}=\left(1+N_{f} / 6\right) g^{2} T^{2}, \quad \lambda_{E}=\frac{9-N_{f}}{12 \pi^{2}} g^{4} T,
$$

though $\lambda_{E}$ starts to contribute to the pressure only at order $g^{6}$. At this order, however, the self-interactions of the massless magnetostatic gluons start to contribute, but these contributions are inherently non-perturbative because the three-dimensional theory for the zero modes $A_{i}(\vec{x})$ is a confining theory! 717273

The thermal pressure of the 4-dimensional theory can be decomposed into contributions from the hard modes $\sim T$, calculable by standard perturbation theory, and soft contributions governed by (79) which involves both perturbatively calculable contributions up to order $g^{5} T^{4}$ and the nonperturbative ones starting at order $g^{6} T^{4}$.

In Ref. 43 the effective theory based on (79) has been used to organize and reproduce the perturbative calculation of the thermal pressure (77) of Refs. 4142. This turns out to be particularly elegant when dimensional regularization is used to provide both the UV and IR cutoffs of the original and effective field theories. as 43

To order $g^{4}$, the contribution of the hard modes can then be written

$$
\begin{aligned}
P_{\text {hard }}=\frac{8 \pi^{2}}{45} & T^{4}\left\{\left(1+\frac{21}{32} N_{f}\right)+\frac{-15}{4}\left(1+\frac{5}{12} N_{f}\right) \frac{\alpha_{s}}{\pi}\right. \\
+ & \left\{244.9+17.24 N_{f}-0.415 N_{f}^{2}+135\left(1+\frac{N_{f}}{6}\right) \ln \frac{\bar{\mu}}{2 \pi T}\right. \\
& \left.\left.-\frac{165}{8}\left(1+\frac{5}{12} N_{f}\right)\left(1-\frac{2}{33} N_{f}\right) \ln \frac{\bar{\mu}}{2 \pi T}\right\}\left(\frac{\alpha_{s}}{\pi}\right)^{2}\right\} .
\end{aligned}
$$

In the first logarithm the dimensional regularization scale $\bar{\mu}$ is associated with regularization in the infrared and thus has to match a similar logarithm in the effective theory, whereas the second logarithm is from UV and involves the first coefficient of the beta function. 
Indeed, calculating the pressure contribution of the soft sector described by (79) in dimensional regularization gives, to three-loop order (neglecting $\lambda_{E}$-contributions)

$$
\begin{aligned}
P_{\text {soft }} / T= & \frac{2}{3 \pi} m_{E}^{3}-\frac{3}{8 \pi^{2}}\left(4 \ln \frac{\bar{\mu}}{2 m_{E}}+3\right) g_{E}^{2} m_{E}^{2} \\
& -\frac{9}{8 \pi^{3}}\left(\frac{89}{24}-\frac{11}{6} \ln 2+\frac{1}{6} \pi^{2}\right) g_{E}^{4} m_{E} .
\end{aligned}
$$

All the contributions to the pressure involving odd powers of $g$ in (77) (as well as part of those involving even powers) are coming from the soft sector. Inserting the leading-order value (80) for $m_{E}$ gives the QCD pressure up to and including order $g^{4} \ln g$; to obtain all the terms to order $g^{5}$, next-toleading order corrections to the $m_{E}$-parameter have to be obtained by a matching calculation as given in Ref. 43 .

In order to obtain the $g^{6} \ln g$-contribution (78) one also needs $g_{E}^{2}$ to order $g^{4}$ (given in Ref. 70 ) and above all the four-loop contribution of the effective theory (79) which has recently been calculated analytically as 63

$$
P_{\mathrm{soft}}^{(4)} / T=N_{g} \frac{\left(N g_{E}^{2}\right)^{3}}{(4 \pi)^{4}}\left(\left[\frac{43}{12}-\frac{157 \pi^{2}}{768}\right] \ln \frac{\bar{\mu}}{g_{E}^{2}}+\left[\frac{43}{4}-\frac{491 \pi^{2}}{768}\right] \ln \frac{\bar{\mu}}{m_{E}}+c\right)
$$

up to a constant $c$ that is strictly nonperturbative and needs to be determined by three-dimensional lattice calculations. Such calculations have been carried out recently 17 , but they depend on an as yet undetermined 4loop matching coefficient. At the moment the conclusion is that it is at least not excluded that the lattice results based on dimensional reduction can be matched to the full four-dimensional results at temperatures of a few times the transition temperature. For this reason, the most reliable results on the thermodynamics of hot QCD (particularly for pure-glue ${ }^{\mathrm{h}} \mathrm{QCD}$ ) remain to date the four-dimensional lattice data.

An interesting feature of the perturbative calculation when organized through the dimensionally reduced effective theory (79) is that the large scale dependences of strict perturbation theory can be reduced when the effective parameters are not subsequently expanded out when their perturbative results are inserted in 826374 . What is more, considering successively the full two-loop and full three-loop contributions from the soft

${ }^{\mathrm{h}}$ Inclusion of fermions is particularly easy in the dimensional reduction method, which makes a full three-dimensional prediction clearly most desirable. 
sector, the results do not exceed the ideal-gas result (as the strictly perturbative results to order $g^{3}$ and $g^{4}$ do), and their scale dependence diminishes by going from two to three-loop order.74

At three-loop order, it is in fact possible to eliminate the scale dependence altogether by a principle of minimal sensitivity. The result in fact agrees remarkably well with the 4 -d lattice results down to $\sim 2.5 T_{c}$. (This is also true when the four-loop logarithms are included provided a suitable value for the unknown constant $c$ in (83) is chosen. 6375 )

While this goes only minimally beyond a strictly perturbative treatment, it strongly suggests that perturbative QCD at high temperature, when supplemented by appropriate resummation of soft physics, is capable of quantitative predictions at temperatures of interest.

\section{Thermodynamics of HTL quasiparticles in QCD}

\subsection{HTL-screened perturbation theory}

In the simple example of scalar $\phi^{4}$ theory, we have seen that a promising strategy for improving the poor convergence of perturbation theory may be screened perturbation theory based on the reorganization of the Lagrangian according to (37). The corresponding resummation changes the UV structure of theory at any finite order of perturbation theory, but removing the additional UV divergences by some minimal subtraction does seem to lead to results which avoid the bad behaviour of conventional perturbation theory.

This method can be extended to QCD by employing the gauge invari-

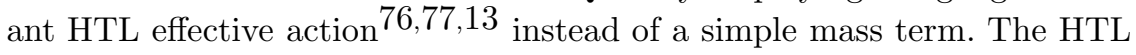
effective action is nonlocal and involves nontrivial vertices in addition to self-energies, but it is uniquely determined by only one mass parameter for gluons (and a further one for quarks, if any)217815960. This variant of screened perturbation theory has been termed HTL perturbation theory (HTLPT) $)^{\mathrm{i}}$ by their authors, but it should be noticed that it is meant to transcend perturbative $H T L$ resummation, where hard propagators and vertices with hard propagators attached remain undressed 9 .

In HTLPT the QCD Lagrangian is improved by writing

$$
\mathcal{L}_{\mathrm{QCD}}=\left.\mathcal{L}_{\mathrm{QCD}}\right|_{\alpha_{s} \rightarrow \delta \alpha_{s}}+(1-\delta) \mathcal{L}_{\mathrm{HTL}}
$$

${ }^{\mathrm{i}}$ We would suggest however to spell this out as HTL-screened perturbation theorv to recall that this method transcends the original HTL resummed perturbation theory 9 . 
with the standard HTL Lagrangian17677113

$$
\mathcal{L}_{\mathrm{HTL}}=-\frac{1}{2} m_{D}^{2} \operatorname{tr} F_{\mu \alpha}\left\langle\frac{v^{\alpha} v^{\beta}}{(v \cdot D(A))^{2}}\right\rangle F^{\mu}{ }_{\beta}+i M^{2} \bar{\psi} \gamma^{\mu}\left\langle\frac{v_{\mu}}{v \cdot D(A)}\right\rangle \psi,
$$

where $v^{\mu}=(1, \vec{v})$ is a light-like four-vector and $\langle\ldots\rangle$ represents the average over the directions $\vec{v}$. Since this only serves as gauge invariant generalization of the thermal mass term for SPT, i.e. a variationally improved perturbation theory, the parameters $m_{D}$ and $M$ need no longer be restricted to their leading-order HTL values (given in eqs. (91) and (94) below). Technically, HTLPT corresponds to using $\delta$ as an expansion parameter that is set equal to 1 eventually.

In Ref. 21, HTLPT has been used to calculate the pressure of QCD at one-loop level. This corresponds to calculating the thermal pressure of a modified free Lagrangian (84) with $\delta=0$. If (but only if) the mass parameters $m_{D}$ and $M$ are identified with their leading-order HTL values (91) and (94), this includes the plasmon term $\sim g^{3}$ of the pressure correctly, but over-includes the leading-order interaction term $\sim g^{2}$ by a factor of 2 . (In Ref. 21 the over-inclusion factor was in fact larger because of an inconsistent usage of dimensional regularization 25 . Corrected numerical results at one-loop level are contained in Refs. 5960 .)

Starting at two-loop order, it becomes possible to determine $m_{D}$ and $M$ by a variational principle. The two-loop pressure in HTLPT is in fact almost prohibitively complicated because of the necessity to include the non-local HTL vertices along with the nontrivial HTL propagators. Refs. ${ }^{5960}$ have simplified this task by expanding the resulting sum-integrals in powers of $m_{D} / T$ and $M / T$ such as to include all terms through order $g^{5}$ if $m_{D} / T$ and $M / T$ are taken to be of order $g$. (These mass expansions have been checked to be fairly accurate in the applications of interest ${ }^{79}$.) When determining $m_{D} / T$ and $M / T$ through variational equations, the two-loop HTLPT result is equivalent with the perturbative result (77) through order $g^{4} \log (g)$, with deviations setting in at order $g^{4}$. The thermal mass parameter $m_{D}$ coincides with the perturbative result for the Debye mass at leading order, though not beyond, which is not too surprising since it is just a variational parameter and not directly related to the full electrostatic propagator; the quark mass parameter $M$ when determined by a variational principle comes out as $\sim g$ at weak coupling, however with a coefficient that differs from leading-order perturbation theory and is in fact subtraction-scale dependent $\underline{60}$.

When evaluated numerically, the HTLPT results, like those of SPT in the scalar case, turn out to be a clear improvement over the conventional 


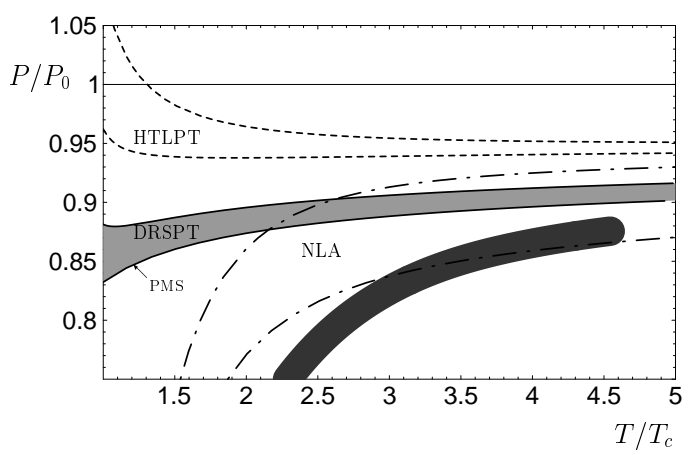

Fig. 12. The two-loop DRSPT result 74 for pure-glue QCD (gray band) in comparison with the two-loop HTLPT result of Ref. 59 (dashed lines) and the "NLA" result of Ref. 25 described in Sect. 4.4 (dash-dotted lines), all with $\bar{\mu}$ varied around $2 \pi T$ by a factor of 2 .

perturbative results in that (at least for all $T \gtrsim 1.5 T_{c}$ ) both the one-loop and the two-loop results are lower than the ideal-gas result while including the plasmon effect $\sim g^{3}$ which in conventional perturbation theory (see Fig. 11) leads to results much above the ideal-gas value in contradiction with all lattice results. Moreover, the one-loop and the two-loop results do not deviate too strongly from each other so that they seem to have satisfactory convergence. On the other hand, they fall short of describing lattice results quantitatively but remain too close to the ideal-gas value and account for only about half of the deviation of the lattice results from the latter.

A hint on the possible reason for this apparent deficiency of HTLPT in the application to the thermodynamics of QCD comes from a simpler variant of SPT, namely when the latter is implemented only in the dimensionally reduced sector (79) by rewriting (79) trivially as

$\mathcal{L}_{\mathrm{EQCD}}=\frac{1}{4} F_{i j}^{a} F_{i j}^{a}+\frac{1}{2}\left(D_{i} A_{0}\right)^{a}\left(D_{i} A_{0}\right)^{a}+\frac{1}{2}\left(m_{E}^{2}+\delta m^{2}\right) A_{0}^{a} A_{0}^{a}-\frac{1}{2} \delta m^{2} A_{0}^{a} A_{0}^{a} .(86$

Here $m_{E}^{2}$ is determined by perturbative matching, whereas the additional $\delta m^{2}$ is to be fixed by a gap equation (i.e., a principle of minimal sensitivity). Like any SPT, this dimensionally reduced SPT (DRSPT) introduces additional UV singularities at finite loop order, which should be minimally subtracted to allow comparison with HTLPT.

Like HTLPT, this formalism is gauge invariant, but very much simpler and it avoids the intermediate modification of hard modes (where ordinary 


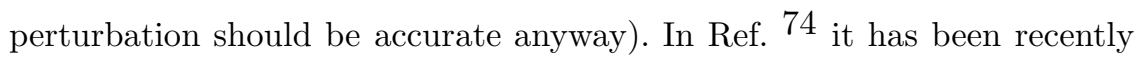
shown that the two-loop result of DRSPT is much closer to the lattice results (reproduced in Fig. 12). Because fermions are integrated out in DRSPT, this also avoids the problem HTLPT appears to have with a gap equation for the fermionic mass. (On the other hand and in contrast to HTLPT, DRSPT is clearly restricted to static quantities.)

Unfortunately, at three-loop order DRSPT gives rise to a gap equation for $\delta m^{2}$ which does not connect to perturbation theory any longer so that it cannot be viewed as an improvement of perturbation theory! 74

\subsection{PI formalism in gauge theories}

In the following we shall describe a different approach in which the 2PI formalism in terms of full propagators is used to construct approximations which avoid the UV problems and ambiguities of SPT and HTLPT. This turns out to amount, at the two-loop level, to a description of the thermodynamics of hot QCD in terms of weakly interacting quasiparticles, whose nontrivial spectral data serve to incorporate (at least in leading and subleading orders) the strong interactions of the elementary degrees of freedom.

In QCD, the thermodynamic potential is a functional of the full gluon $(D)$, quark $(S)$, and Faddeev-Popov ghost $\left(D_{g h}\right)$ propagators,

$$
\begin{aligned}
\beta \Omega\left[D, S, D_{g h}\right]= & \frac{1}{2} \operatorname{Tr} \log D^{-1}-\operatorname{Tr} \log S^{-1}-\operatorname{Tr} \log D_{g h}^{-1} \\
& -\frac{1}{2} \operatorname{Tr} \Pi D+\operatorname{Tr} \Sigma S+\operatorname{Tr} \Pi_{g h} D_{g h}+\Phi\left[D, S, D_{g h}\right],
\end{aligned}
$$

where Tr now includes traces over color indices, and also over Lorentz and spinor indices when applicable. The self-energies for gluons, quarks and ghosts are denoted by $\Pi, \Sigma$ and $\Pi_{g h}$, respectively.

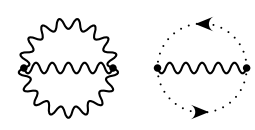

(a) (b)

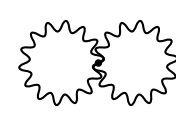

(c)

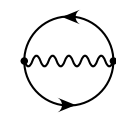

(d)

Fig. 13. Diagrams for $\Phi$ at 2-loop order in QCD. Wiggly, plain, and dotted lines refer respectively to gluons, quarks, and ghosts.

In the following we shall consider a two-loop $\Phi$-derivable approximation which amounts to keeping the diagrams displayed in Fig. 13] This again 
leads to the important simplification that the entropy is given by a comparatively simple expression

$$
\begin{aligned}
\mathcal{S}= & -\operatorname{tr} \int \frac{d^{4} k}{(2 \pi)^{4}} \frac{\partial n(\omega)}{\partial T}\left[\operatorname{Im} \log D^{-1}-\operatorname{Im} \Pi \operatorname{Re} D\right] \\
& -2 \operatorname{tr} \int \frac{d^{4} k}{(2 \pi)^{4}} \frac{\partial f(\omega)}{\partial T}\left[\operatorname{Im} \log S^{-1}-\operatorname{Im} \Sigma \operatorname{Re} S\right],
\end{aligned}
$$

with vanishing interaction term $\mathcal{S}^{\prime}$ (cf. (69)), and this also holds true for fermion number densities:

$$
\mathcal{N}=-2 \operatorname{tr} \int \frac{d^{4} k}{(2 \pi)^{4}} \frac{\partial f(\omega)}{\partial \mu}\left[\operatorname{Im} \log S^{-1}-\operatorname{Im} \Sigma \operatorname{Re} S\right],
$$

where the completely analogous interaction term $\mathcal{N}^{\prime}$ is equally absent at the two-loop level.

While $\Phi$-derivable approximations are symmetry preserving as concerns global symmetries 22 , local gauge invariance is not respected, so this approximation introduces gauge dependences as long as vertices are not dressed in a self-consistent manner in line with the self-energies ${ }^{j}$. These gauge dependences are actually parametrically suppressed at the stationary point 81 , where they enter only at twice the order of the truncation. However, in what follows we shall construct further approximations based on the gauge invariant hard thermal loops, and this will entail manifest gauge independence in our applications. In these approximations, where the self-consistency of the two-loop $\Phi$-derivable scheme is only approximately realized, but such that the deviations from full self-consistency are of the order of three-loop corrections, only the two physical polarizations of the gluons contribute and the Faddeev-Popov ghosts just cancel the unphysical gauge degrees of freedom of the former so that they can be simply dropped.

\subsection{Approximately self-consistent entropy and quark density}

As discussed above, conventional thermal perturbation theory exhibits very poor apparent convergence as soon as collective phenomena such as the plasmon effect are included, which involve odd powers in the coupling. The self-consistent entropy and density functionals at two-loop order also contain the plasmon effect, but together with higher-order terms that ordinarily would have been discarded by a strictly perturbative expansion in $g$ because

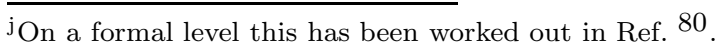


otherwise the renormalization programme could not have been carried out. By contrast, the two-loop entropy and density functionals (88) and (89) are UV finite expressions and can be evaluated in a nonperturbative manner using the high-temperature/density approximation of the gluon and quark propagators, which are finite at leading and next-to-leading order in HTL perturbation theory.

\subsubsection{HTL approximation}

As a first approximation we consider the two-loop entropy and density functionals evaluated completely using HTL (HDL) propagators. From the discussion of the scalar model we anticipate that this includes correctly the leading-order interaction contribution $\propto g^{2}$ in the entropy (in contrast to the pressure). ${ }^{\mathrm{k}}$

But unlike the scalar $\phi^{4}$ model, where the HTL self-energy is a simple constant mass, cf. (18), the HTL self-energies for gluons and quarks contain two non-local structure functions each. The gluon HTL self-energy contains transverse and longitudinal components given by

$$
\begin{aligned}
\hat{\Pi}_{L}(\omega, k) & =\hat{m}_{D}^{2}\left[1-\frac{\omega}{2 k} \log \frac{\omega+k}{\omega-k}\right], \\
\hat{\Pi}_{T}(\omega, k) & =\frac{1}{2}\left[\hat{m}_{D}^{2}+\frac{\omega^{2}-k^{2}}{k^{2}} \hat{\Pi}_{L}\right],
\end{aligned}
$$

where

$$
\hat{m}_{D}^{2}=\left(2 N+N_{f}\right) \frac{g^{2} T^{2}}{6}+N_{f} \frac{g^{2} \mu^{2}}{2 \pi^{2}}
$$

is the (leading-order) Debye screening mass. ${ }^{1}$ As is well knowr 8384 , the gluon propagator dressed by these self-energies has quasiparticle poles at $\omega_{T, L}(k)$ with momentum-dependent effective masses and Landau damping cuts for $|\omega|<k$. When $k \gg \hat{m}_{D}$, the pole corresponding to the collective longitudinal excitation has exponentially vanishing residue $\stackrel{85}{\text {, whereas }}$

${ }_{\mathrm{k}} \mathrm{A}$ different procedure for including the full HTL propagators has been proposed by Peshiel 82 , who considers a (somewhat ad-hoc) modification of the $\Phi$ functional such as to have correct leading-order contributions and UV finiteness when full propagators are approximated by HTL ones. This turns out to be equivalent to our results when expanded out to order $g^{3}$.

${ }^{1}$ For simplicity we shall write out our formulae for only one value of the chemical potential; the generalization to several different chemical potentials is straightforward. 
transverse excitations tend to $\omega \rightarrow \sqrt{k^{2}+m_{\infty}^{2}}$, where the asymptotic mass is given by

$$
m_{\infty}^{2}=\hat{\Pi}_{T}(k, k)=\frac{1}{2} \hat{m}_{D}^{2} .
$$

The (massless) quark propagator at finite temperature or density is split into two separate branches of opposite ratio of chirality over helicity with propagators $\Delta_{ \pm}=\left[-\omega+k \pm \Sigma_{ \pm}\right]^{-1}$. In the HTL approximation, the respective self-energies read $\underline{86}$.

$$
\hat{\Sigma}_{ \pm}(\omega, k)=\frac{\hat{M}^{2}}{k}\left(1-\frac{\omega \mp k}{2 k} \log \frac{\omega+k}{\omega-k}\right),
$$

where $\left(C_{f}=\left(N^{2}-1\right) / 2 N\right)$

$$
\hat{M}^{2}=\frac{g^{2} C_{f}}{8}\left(T^{2}+\frac{\mu^{2}}{\pi^{2}}\right) .
$$

The dressed fermion propagators have quasiparticle poles at $\omega_{ \pm}(k)$ and Landau damping cuts. At large $k$ and positive frequency $\omega_{+} \rightarrow \sqrt{k^{2}+M_{\infty}^{2}}$ with asymptotic mass $M_{\infty}^{2}=2 \hat{M}^{2}$; the additional branch $\omega_{-}$which has no analogue at zero temperature and density has exponentially vanishing residue 8788 .

Although the various HTL self-energies constitute a leading-order result only under the condition of soft momenta and frequencies, the result for the asymptotic masses applies also for hard momenta 89190 .

Using these expressions in the entropy formula (88) this takes care of all contributions of order $g^{2}$, but only part $(\leq 1 / 4)$ of the plasmon term $\sim g^{3}$. However, it also contains infinitely many higher-order terms which despite being incomplete may help to get rid of the pathological behaviour of the perturbation series truncated at low orders in $g$. Among such higher-order contributions is for instance a $g^{4}$ contribution reading (for pure glue)

$$
\mathcal{S}_{H T L}^{(4)}=-N_{g} \frac{\hat{m}_{D}^{4}}{16 \pi^{2} T}\left(\log \frac{T}{\hat{m}_{D}}+1.55 \ldots\right)
$$

involving a $g^{4} \log (1 / g)$-term, whose coefficient in pure-glue QCD is $1 / 12$ of that of the complete perturbative result. ${ }^{\mathrm{m}}$ By contrast, simple massive quasiparticle models such as those used in Refs. 2728 do not have a $g^{4} \log (1 / g)$-term in the entropy at all.

${ }^{\mathrm{m}}$ The correct coefficient will be restored by $O\left(g^{4} \log (1 / g) T^{2}\right)$ corrections to $m_{\infty}^{2}$, whereas the constant under the logarithm also receives three-loop contributions. 


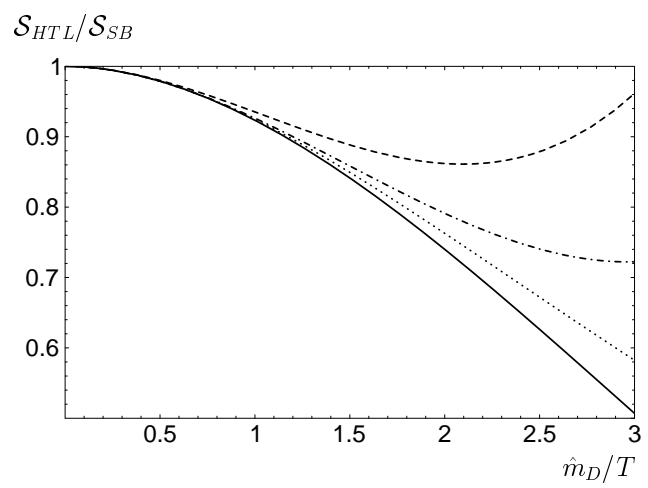

Fig. 14. HTL entropy per gluonic degree of freedom normalized to its Stefan-Boltzmann value as a function of the Debye mass $\hat{m}_{D}(T, \mu) / T$. The full line gives the complete numerical result; the dashed line corresponds to the perturbative result to order $\left(\hat{m}_{D} / T\right)^{3} \sim g^{3}$. The dotted line gives the entropy for scalar degrees of freedom with momentum-independent mass $m=m_{\infty}=\hat{m}_{D} / \sqrt{2}$; its perturbative approximant is given by the dash-dotted line.

In Fig. 14 the numerical result for $\mathcal{S}_{H T L} / \mathcal{S}_{S B}$ in the case of pure glue is given as a function of $\hat{m}_{D} / T$, which is the only independent parameter. The HTL result is depicted by the full line and is found to be a monotonically decreasing function of $\hat{m}_{D} / T$. If this were expanded in powers of $\hat{m}_{D} / T$ and truncated beyond $\left(\hat{m}_{D} / T\right)^{3} \sim g^{3}$ (dashed line in Fig. 14), this property would have been lost at $\hat{m}_{D} /(2 \pi T) \approx 1 / 3$, where one might still expect a sufficiently clear separation of hard and soft scales which is a prerequisite of the HTL approximation.

The numerical result for $\mathcal{S}_{H T L}$ is in fact very close to a simple massive quasiparticle model with entropy $2 N_{g} \mathcal{S}_{S B}\left(m_{\infty}\right)$, represented by the dotted line in Fig. 14 This is due to large cancellations between the longitudinal and transverse contributions to the HTL entropy. However, quite remarkably one has $\mathcal{S}_{H T L}<2 N_{g} \mathcal{S}_{S B}\left(m_{\infty}\right)$ even though the latter contains $30 \%$ less of the plasmon term $\sim g^{3}$, which would be expected to work in the opposite direction. This is further emphasized by comparison with its perturbative approximation given by the dash-dotted line.

In Fig. 15 the HTL entropy of 1 quark degree of freedom at zero chemical potential is displayed as a function of the fermionic plasma frequency $\hat{M}$. Like in the pure-glue case, this contains also an (incomplete) $g^{4} \log (1 / g)$ 
contributions that is not present in simpler quasiparticle models:

$$
\mathcal{S}_{f, H T L}^{(4)}=N N_{f} \frac{\hat{M}^{4}}{\pi^{2} T}\left(\log \frac{T}{\hat{M}}+0.22 \ldots\right)
$$

$\mathcal{S}_{f, H T L}$ does not contain any contributions to the plasmon term $\sim g^{3}$, which entirely come from NLO corrections to $M_{\infty}$. There is therefore rather little deviation of the full numerical result for the fermionic contribution (solid line) from the perturbative one truncated above order $g^{2}$ (dashed line).

Compared to the entropy of a simple massive fermionic quasiparticle model $\mathcal{S}_{f}^{(0)}\left(M_{\infty}\right)$ (dotted line in Fig. 15), one finds extremely good numerical agreement, which again takes place only after adding up all the quasiparticle $((+)$ and $(-))$ and Landau-damping contributions, however.

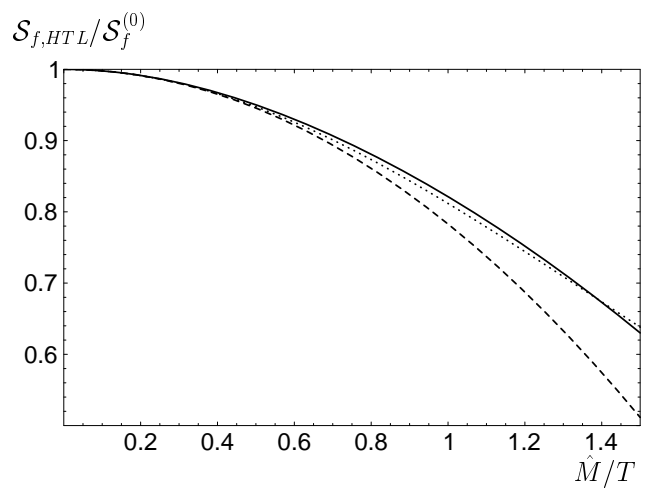

Fig. 15. HTL entropy per quark degree of freedom at $\mu=0$ normalized to its free value (solid line), the corresponding perturbative order- $g^{2}$ result (dashed line), and the entropy of a quark with constant mass $M_{\infty}$ (dotted line).

In the case of zero temperature but high chemical potential, the quantity of interest is the quark density. This does not contain any plasmon term $\sim g^{3}$, but rather $\sim g^{4} \log (1 / g)$. The HDL approximation does contain some though not all of this term:

$$
\mathcal{N}_{H D L}^{(4)}=N N_{f} \frac{\hat{M}^{4}}{\pi^{2} \mu}\left(\log \frac{\mu}{\hat{M}}+0.35 \ldots\right)
$$

Order- $g^{2} \log g$ contributions to the asymptotic masses of the quark and gluon quasiparticles, still within the framework of the 2-loop $\Phi$-derivable approximation, are responsible for the remaining contribution to the coefficient of the $g^{4} \log g$-term, while the coefficient under the logarithm also receives 3-loop contributions. 
In Fig. 16 the numerical result for $\mathcal{N}_{H D L}$ is given as a function of $\hat{M} / \mu$ (full line) and compared to that of a simple quasiparticle model

$$
\left.\mathcal{N}_{0}\left(M_{\infty}\right)\right|_{T=0}=\frac{1}{3 \pi^{2}}\left(\mu^{2}-M_{\infty}^{2}\right)^{\frac{3}{2}} \theta\left(\mu-M_{\infty}\right)
$$

as well as to a perturbative approximation truncated beyond $(\hat{M} / \mu)^{2} \sim g^{2}$. Remarkably, the full HDL result drops to zero at almost the same value as the simple quasiparticle model. However, the former becomes negative thereafter, showing that the HDL approximation is breaking down at $M_{\infty} / \mu>1$ at the latest.

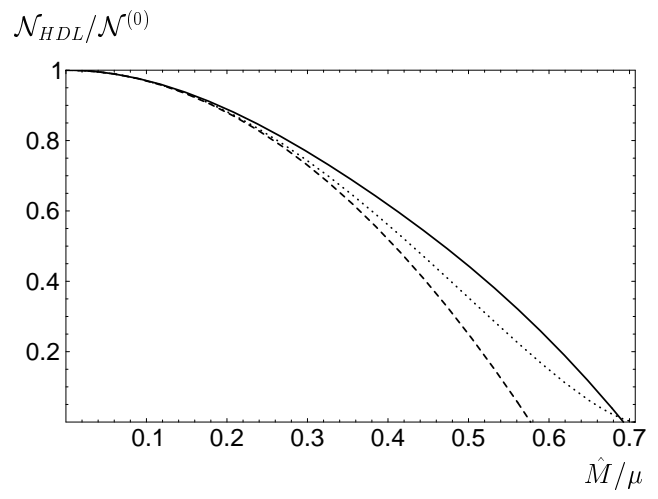

Fig. 16. HDL quark density at $T=0$ normalized to its free value (solid line), the corresponding perturbative order- $g^{2}$ result (dashed line), and the free quark density of a quark with constant mass $M_{\infty}$ (dotted line).

\subsubsection{Next-to-leading approximations}

The plasmon term $\sim g^{3}$ at high temperatures $T \gg \hat{m}_{D}$ becomes complete only after inclusion of the next-to-leading correction to the asymptotic thermal masses $m_{\infty}$ and $M_{\infty}$. These are determined in standard HTL perturbation theory through

$$
\begin{aligned}
& \delta m_{\infty}^{2}(k)=\operatorname{Re} \delta \Pi_{T}(\omega=k)
\end{aligned}
$$

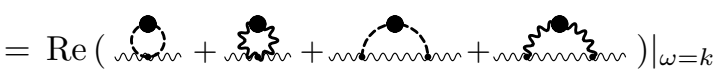


where thick dashed and wiggly lines with a blob represent HTL propagators for longitudinal and transverse polarizations, respectively. Similarly,

$$
\begin{aligned}
& \frac{1}{2 k} \delta M_{\infty}^{2}(k)=\delta \Sigma_{+}(\omega=k)
\end{aligned}
$$

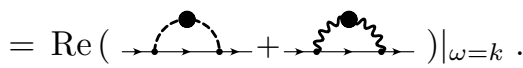

The explicit proof that these contributions indeed restore the correct plasmon term is given in Ref. 25 .

These corrections to the asymptotic thermal masses are, in contrast to the latter, nontrivial functions of the momentum, which can be evaluated only numerically. However, as far as the generation of the plasmon term is concerned, these functions contribute in the averaged form

$$
\bar{\delta} m_{\infty}^{2}=\frac{\int d k k n_{\mathrm{BE}}^{\prime}(k) \operatorname{Re} \delta \Pi_{T}(\omega=k)}{\int d k k n_{\mathrm{BE}}^{\prime}(k)}
$$

and similarly

$$
\bar{\delta} M_{\infty}^{2}=\frac{\int d k k n_{\mathrm{FD}}^{\prime}(k) \operatorname{Re} 2 k \delta \Sigma_{+}(\omega=k)}{\int d k k n_{\mathrm{FD}}^{\prime}(k)} .
$$

which can be determined analytically 24 , leading to the remarkably simple expressions

$$
\begin{aligned}
\bar{\delta} m_{\infty}^{2} & =-\frac{1}{2 \pi} g^{2} N T \hat{m}_{D}, \\
\bar{\delta} M_{\infty}^{2} & =-\frac{1}{2 \pi} g^{2} C_{f} T \hat{m}_{D}, C_{f}=N_{g} /(2 N),
\end{aligned}
$$

These corrections only pertain to the hard excitations; in contrast to the scalar case, in gauge theories the one-loop corrections to the various thermal masses of soft excitations are known to differ substantially from (103) or (104). For instance, the gluonic plasma frequency at $k=0$ reads $\frac{91}{\delta} m_{p l .}^{2} / \hat{m}_{p l .}^{2} \approx-0.18 \sqrt{N} g$, which is only about a third of $\bar{\delta} m_{\infty}^{2} / m_{\infty}^{2}$; the NLO correction to the nonabelian Debye mass on the other hand is even positive for small coupling and moreover logarithmically enhanced 39 $\delta m_{D}^{2} / \hat{m}_{D}^{2}=+\sqrt{3 N} /(2 \pi) \times g \log (1 / g)$.

For an estimate of the effects of a proper incorporation of the next-toleading order corrections we have therefore proposed to include the latter only for hard excitations and to define our next-to-leading approximation (for gluons) through

$$
\mathcal{S}_{N L A}=\left.\mathcal{S}_{H T L}\right|_{\text {soft }}+\left.\mathcal{S}_{\bar{m}_{\infty}^{2}}\right|_{\text {hard }},
$$


where $\bar{m}_{\infty}^{2}$ includes (103). To separate soft $\left(k \sim \hat{m}_{D}\right)$ and hard $(k \sim 2 \pi T)$ momentum scales, we introduce the intermediate scale $\Lambda=\sqrt{2 \pi T \hat{m}_{D} c_{\Lambda}}$ and consider a variation of $c_{\Lambda}=\frac{1}{2} \ldots 2$ as part of our theoretical uncertainty.

Another crucial issue concerns the definition of the corrected asymptotic mass $\bar{m}_{\infty}$. For the range of coupling constants of interest $(g>1)$, the correction $\left|\bar{\delta} m_{\infty}^{2}\right|$ is greater than the LO value $m_{\infty}^{2}$, leading to tachyonic masses if included in a strictly perturbative manner.

In the simple scalar model of Sect. 2.3.2 we have seen that the requirement of self-consistency for the soft modes leads to a quadratic gap equation for the asymptotic thermal mass, eq. (76).

In QCD, where the (non-local) gap equations are much too complicated to be attacked directly, we simply consider perturbatively equivalent expressions for the corrected $\bar{m}_{\infty}$ which are monotonic functions in $g$. Besides the solution to a quadratic equation analogous to (76), namely

$$
\bar{m}_{\infty}^{2}=\frac{g^{2}\left(N+N_{f} / 2\right) T^{2}}{6}-\frac{g^{2} N T}{\sqrt{2} \pi} \bar{m}_{\infty}
$$

we have tried the simplest Padé approximant (degree $[2,1]$ ), which also gives a greatly improved approximation to the solution of the gap equation of the scalar (large $N$ ) model. In QCD, our final results do not depend too much on whether we use the Padé approximant $\underline{2324}$ or a quadratic gap equation 25 .

The main uncertainty rather comes from the choice of the renormalization scale which determines the magnitude of the running strong coupling constant (chosen to satisfy the renormalization group equation to 2-loop order in the following).

In Fig. 17 the numerical results for the HTL entropy and the NLA one are given as a function of $T / T_{c}$ with $T_{c}$ chosen as $T_{c}=1.14 \Lambda_{\overline{\mathrm{MS}}}$. The full lines show the range of results for $\mathcal{S}_{H T L}$ when the renormalization scale $\bar{\mu}$ is varied from $\pi T$ to $4 \pi T$; the dash-dotted lines mark the corresponding results for $\mathcal{S}_{N L A}$ with the additional variation of $c_{\Lambda}$ from $1 / 2$ to 2 . The

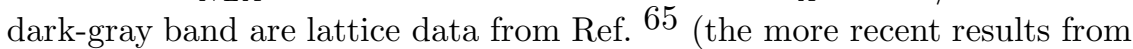
Ref. 66 are consistent with the former within error bars and centered around the upper boundary of the gray band for $T \approx 3 T_{c}$ and somewhat flatter around $2 T_{c}$ ). Evidently, there is very good agreement for $T>2.5 T_{c}$.

When $N_{f}>0$ massless quarks are included, the next-to-leading corrections to the asymptotic fermionic masses (104) have the same problem as the bosonic ones that for large coupling the asymptotic masses may turn tachy- 


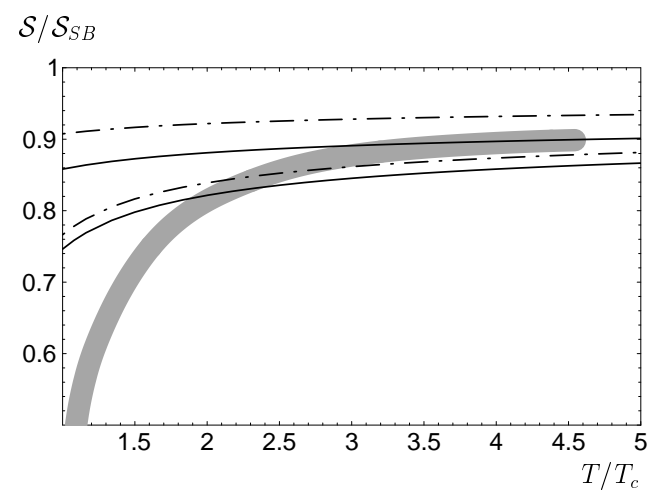

Fig. 17. Comparison of the lattice data for the entropy of pure-glue SU(3) gauge theory of Ref. 65 (gray band) with the range of $\mathcal{S}_{H T L}$ (solid lines) and $\mathcal{S}_{N L A}$ (dash-dotted lines) for $\bar{\mu}=\pi T \ldots 4 \pi T$ and $c_{\Lambda}=1 / 2 \ldots 2$.

onic. In Ref. 25 they have therefore been treated like the bosonic asymptotic masses, by postulating the simplest quadratic gap equation which is perturbatively equivalent to (104). However, there is an obvious asymmetry in that only gluonic quantities appear on the right-hand side of (1031) and (104). Indeed, treating them in the same manner is not consistent in the limit of $N_{f} \rightarrow \infty$, which has been solved recently in Refs. 92 . To be consistent with this limit, the fermionic gap equation has to remain linear as it is in perturbation theory. We therefore restrict our requirement of a perturbative equivalent gap equation with monotonic behaviour only to the bosonic (gluonic) sector. For the fermions we proceed by treating the right-hand sides of eqs. (103) and (104) in the same manner, which amounts to 93

$$
\bar{M}_{\infty}^{2}=\frac{g^{2} N_{f} C_{f} T^{2}}{4}-\frac{1}{\sqrt{2} \pi} g^{2} C_{f} T \bar{m}_{\infty} .
$$

There is then no negative feedback from the fermion mass itself, it only inherits higher-order terms from the solution to $\bar{m}_{\infty}$. This does not avoid the appearance of tachyonic masses when both $N_{f}$ and $g$ are large, but it turns out that for $N_{f} \leq 3$ one still has a monotonic behaviour of $M_{\infty}$ as a function of $g$. In fact, in the case $N_{f}=3$ the solutions to the correspondingly corrected gap equations happen to coincide precisely with those of the (structurally rather different) decoupled quadratic equations adopted in Ref. 25 . As a result, the changes on the results of Ref. $\frac{25}{25}$ are rather minimal for phenomenologically interesting values of $N_{f}$. (In the limit $N_{f} \rightarrow \infty$, there are more important effects, such that the picture of stable quasi-particles 


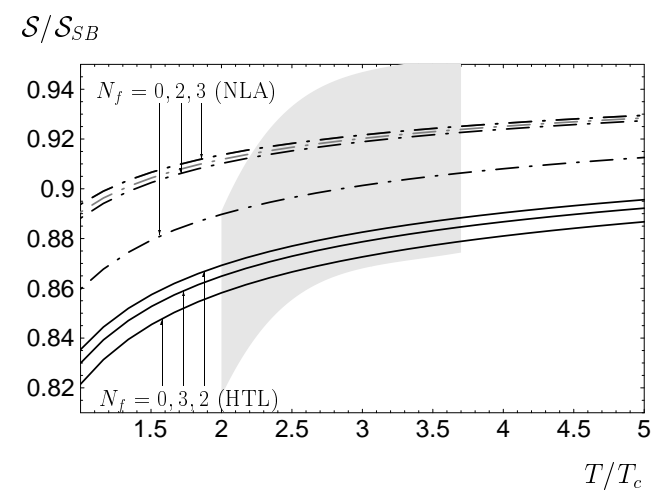

Fig. 18. Comparison of the HTL entropy (solid lines) and the NLA estimate (dashdotted lines) for $N_{f}=0,2,3$ with the estimated continuum extrapolation of $N_{f}=2$ lattice data of Ref. 94. (The gray dash-dotted line corresponds to using a quadratic fermionic gap equation, which only has an effect for NLA $N_{f}=2$, which is minimally higher than the result obtained with a linear one required for consistency with the large$N_{f} \operatorname{limit}(93)$.

seems to have to be abandoned above some large coupling, but up to this point the quantitative agreement with the exact result is quite good 93 .)

In Fig. 18 the (updated) results for the entropy with $N_{f} \leq 3$ massless quarks are compared with a recent estimate $\frac{94}{4}$ of the continuum limit of lattice results for $N_{f}=2$ (gray band), but now with $\mathcal{S}_{H T L}$ and $\mathcal{S}_{N L A}$ evaluated for the central choice of $\bar{\mu}=2 \pi T$ and $c_{\Lambda}=1$ (with unchanged $\left.T_{c} / \Lambda_{\overline{\mathrm{MS}}}\right)$. When $N_{f}$ is increased, there are competing effects of larger thermal masses versus slower running of $\alpha_{s}$, which result into a rather weak dependence of our results on $N_{f}$ as a function of $T / \Lambda_{\overline{\mathrm{MS}}}$ as it is in Fig. 18 This seems to be consistent with the very small $N_{f}$ dependence observed on the lattice (when the thermodynamic potentials are normalized by their ideal-gas limit and expressed as a function of $T / T_{c}$ ).

It should be understood, however, that the simple quadratic gap equation (106) and its extension to the fermionic sector, eq. (107), are at present only an estimate of how the next-to-leading order corrections (99) and (100) in actuality modify the spectral properties of the hard quasiparticles. Moreover, the former are included so far in the averaged form (101) and (102). A possible refinement is to include the non-trivial momentum dependence of (99) and (100) which we expect to render a separation scale between hard and soft quasiparticles superfluous because the corrections (99) and (100) appear to grow with momentum so that their incompleteness at soft 
momenta may be negligible. When doing so we also hope to be able to come to a better understanding of the mechanism which provides the nonlinear feedback preventing tachyonic masses from appearing.

\subsection{Pressure}

In the above, we have concentrated on the entropy (and quark number density) as the thermodynamic potential which is most suitable for a (HTL) quasiparticle description.

Were it not for the trace anomaly ${ }^{95}\left\langle T_{\mu}^{\mu}\right\rangle=\mathcal{E}-3 P \neq 0$ the corresponding pressure would be simply given by $P=(T \mathcal{S}+\mu \mathcal{N}) / 4$ (in the case of massless quarks). The correct relation is instead provided by an integration such as

$$
P(T, \mu)=\int_{T_{1}}^{T} \mathcal{S}\left(T^{\prime}, \mu\right) d T^{\prime}+P\left(T_{1}, \mu\right) .
$$

Up to a single integration constant, entropy and density evidently contain all the relevant information. This integration constant, however, represents strictly nonperturbative information, because it amounts to fixing the bag constant. Since QCD is asymptotically free, but confining at low temperature, this constant cannot be fixed by requiring that the thermal pressure as obtained from entropy and density vanishes at zero temperature. It is also not possible to determine $P\left(T_{1}\right)$ from the requirement that both $p\left(\alpha_{s}\right)=P(T) / P_{0}(T)$ and $s\left(\alpha_{s}\right)=S(T) / S_{0}(T)$ approach 1 in the limit of $\alpha_{s} \rightarrow 0$, because the differential equation $p\left(\alpha_{s}\right)+\frac{1}{4} \beta\left(\alpha_{s}\right) p^{\prime}\left(\alpha_{s}\right)=s\left(\alpha_{s}\right)$ with $\beta\left(\alpha_{s}\right)=-\beta_{0} \alpha_{s}^{2}-\beta_{1} \alpha_{s}^{3}-\ldots$ has as homogeneous solutions

$$
p\left(\alpha_{s}\right)_{\text {hom. }}=C \exp \left\{-\frac{1}{\alpha_{s}}\left[4 \beta_{0}^{-1}+O\left(\alpha_{s}\right)\right]\right\},
$$

and this vanishes at $\alpha_{s}=0$ together with all its derivatives.

Instead we can fix the integration constant by comparison with lattice data for the pressure at $\mu=0$. A simple possibility for our purposes is to take $T_{1}=T_{c}$ and $P\left(T_{c}, 0\right)=0$. For $T$ substantially larger than $T_{c}$ this does not influence much the results derived from entropy and density, since in the ratio $P / P_{0}$ the (bag) constant term decays like $T^{-4}$.

The result obtained from integrating the entropy in the case of pure-glue QCD with boundary condition $P\left(T_{c}\right)=0$ is shown in Fig. 12] and compared with available lattice data as well as the results obtained recently in 2-loop HTLPT by Andersen et al. .59 and in a dimensionally reduced variant of $\mathrm{SPT} 74$ 


\subsubsection{Finite chemical potential}

For finite chemical potential, the entropy and quark density expressions satisfy the Maxwell relations

$$
\left.\frac{\partial \mathcal{S}}{\partial \mu}\right|_{T}=\left.\frac{\partial \mathcal{N}}{\partial T}\right|_{\mu}
$$

only to the same order in $g$ to which they are accurate when expanded out perturbatively.

In simple quasiparticle models 2728 one therefore has to allow for a bag function (instead of a bag constant) to restore thermodynamic consistency. This can in fact be utilized for matching lattice results and mapping them in a phenomenological manner to nonvanishing chemical potentia29, where recently significant progress has been made on the lattice side 96 . This includes by now concrete results on the thermodynamical potentials 97 , which have been found to agree well with the simple quasiparticle models 98 .

A similar programme has been carried through with the HTL expressions for entropy and density in place of those of the simpler quasiparticle models with constant masses, and the results are, like those of the simpler models, encouraging 99. An extension which includes next-to-leading corrections to the asymptotic thermal masses is work in progress.

\subsection{Quark number susceptibilities}

Quark number susceptibilities (QNS) are defined as the second derivatives of the pressure with respect to the chemical potentials of the different quark flavors:

$$
\chi_{i j} \equiv \frac{\partial \mathcal{N}_{i}}{\partial \mu_{j}}=\frac{\partial^{2} P}{\partial \mu_{i} \partial \mu_{j}}=\chi_{j i}
$$

where $i, j$ are flavor indices.

These quantities are of direct interest for studying the transition to the quark-gluon plasma phase as they can be related to fluctuations in conserved charges which can discriminate against a purely hadronic phase 100 .

On the theoretical side, there are new results on QNS from lattice gauge theory 101102103104 , extending them to higher $T / T_{c}$ which can be compared against conventional perturbative results and those resulting from resummation schemes aiming at overcoming the poor convergence of the former. 


\subsubsection{Diagonal quark number susceptibilities}

The diagonal QNS, which for degenerate quark flavors are all equal and denoted simply by $\chi$ in what follows, are found to increase sharply at the deconfinement phase transition toward a large percentage of the ideal gas value $\chi_{0}=N T^{2} / 3$ (for $\mathrm{SU}(N)$ and massless quarks).

In perturbation theory, the series expansion of $\chi / \chi_{0}$ is now known 105 through order $g^{6} \ln (1 / g)$ and the coefficient under the log at order $g^{6}$ is in fact computable in perturbation theory, in contrast to the one appearing in the thermal pressure, though not yet available. The first few terms of this expansion read 1065 .

$$
\begin{aligned}
\frac{\chi}{\chi_{0}}= & 1-\frac{1}{2} \frac{3}{N} \frac{N_{g}}{8}\left(\frac{g}{\pi}\right)^{2}+\frac{3}{N} \frac{N_{g}}{8} \sqrt{\frac{N}{3}+\frac{N_{f}}{6}}\left(\frac{g}{\pi}\right)^{3} \\
& -\frac{3}{4} \frac{N_{g}}{8}\left(\frac{g}{\pi}\right)^{4} \log \frac{1}{g}+\mathcal{O}\left(g^{4}\right)
\end{aligned}
$$

(for the more lengthy results on the subsequent coefficients we refer the reader to Ref. 105 ).

In these strictly perturbative results the plasmon term overcompensates the leading-order interaction term, much like in the case of the pressure, though less severely so (for $N_{f}=2$ the diagonal QNS fall below the idealgas value only when $T>40 T_{c}$, and only for $T>700 T_{c}$ does one find a growth of $\chi / \chi_{0}$ with temperature as observed on the lattice - in the case of the pressure these temperatures below which perturbation theory clearly fails are orders of magnitudes higher).

The $\Phi$-derivable 2-loop expression (89) captures both the leading-order term $\propto g^{2}$ and the plasmon term $\propto g^{3}$ accurately, together with a subset of higher-order terms that are usually expanded out in 2-loop perturbation theory.

As additional approximations we may start from the HTL quark density $\mathcal{N}_{\text {HTL }}$ where (89) is evaluated with HTL propagators. Differentiation with respect to the chemical potential yields the corresponding approximation for the diagonal ${ }^{\mathrm{n}}$ QNS.

While this approximation contains the leading-order term $\propto g^{2}$ accurately, it does not include anything of the plasmon term. In the QNS, the plasmon term arises completely from corrections to the asymptotic thermal masses of quarks according to (100).

$\overline{{ }^{n} \text { In the HTL approximation the QNS }}$ are purely diagonal. 


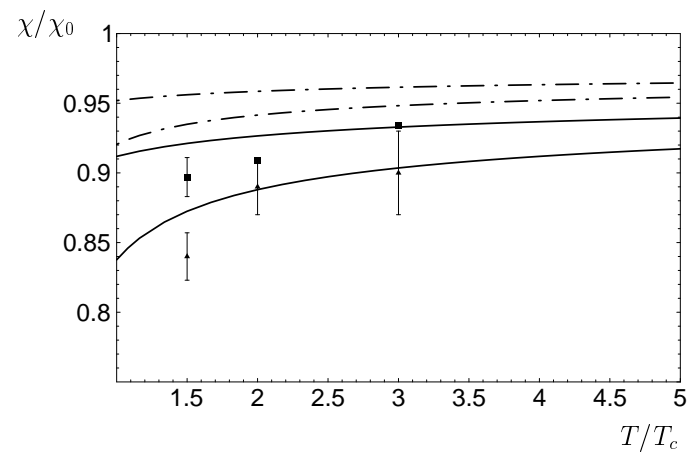

Fig. 19. Comparison of our results for $\chi / \chi_{0}$ in the formal limit $N_{f}=0$ with two recent continuum-extrapolated lattice results for quenced $\mathrm{QCD} 103$.

Numerically, the HTL approximation gives results somewhat above the strictly perturbative result to order $g^{2}$, and, using the same gap equations for the asymptotic thermal masses of quarks as in the previous application, the result is halfway towards the ideal-gas result, whereas a strictly perturbative inclusion of the plasmon effect would have resulted in a result in excess of the free one.

Fig. 19] shows the results for quenched QCD together with recent results for two different continuum extrapolations 103 (both are higher than the previous lattice results $\frac{108}{2}$ ). Fig. 20 shows our prediction 107 for $N_{f}=2$ in comparison with the lattice data of Ref. 101 , for which a continuum extrapolation is not yet available, however.

It would be interesting to compare our predictions with HTLPT to twoloop order; the one-loop HTL-resummed results from charge correlators $\$ 109$ suffer from severe overcounting while missing out the plasmon term 110 so that one should not perform a comparison yet.

\subsubsection{Off-diagonal quark number susceptibilities}

Since lattice results are starting to become available also for the off-diagonal QNS, which in the limit of massless QCD we denote collectively by $\tilde{\chi}$, we briefly discuss this quantity, too. In contrast to the diagonal QNS, the idealgas value of off-diagonal QNS is zero, so this directly probes the interactions in the system.

The leading-order contribution to off-diagonal QNS require two fermion loops connected by gluon lines. The diagram with just one gluon exchange 


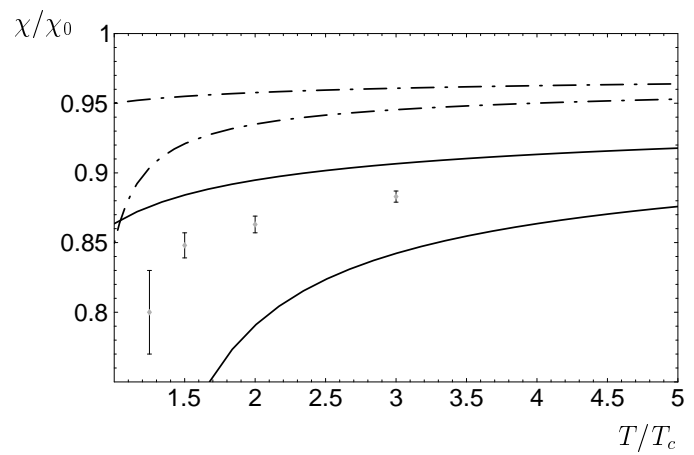

Fig. 20. Comparison of our results for $\chi / \chi_{0}$ in massless $N_{f}=2$ QCD with the lattice results of Ref. 101 (no continuum extrapolation). Full lines refer to the HTL approximation, dash-dotted lines to NLA, when the $\overline{\mathrm{MS}}$ scale is varied from $\bar{\mu}=\pi T$ to $\bar{\mu}=4 \pi T$.

vanishes by colour neutrality. The one with two gluon exchange is non-zero, but because the fermion loops are then even functions of $\mu$, it contributes to $\tilde{\chi}$ only when $\mu \neq 0$, starting at order $g^{3}$, namely

$$
\chi_{i j}=\frac{g^{4}\left(N^{2}-1\right) T \mu_{i} \mu_{j}}{16 \pi^{5} m_{D}} \quad \text { for } i \neq j .
$$

This contribution is indeed contained in the next-to-leading approximation discussed above, but only after the corrections to the asymptotic thermal masses have been included.

When all chemical potentials vanish (which is the case most easily accessible to lattice studies), the above approximations of the QNS become strictly diagonal. In this limit the lowest-order diagram contributing to offdiagonal QNS is the 4-loop "bugblatter" diagram shown in Fig. 21 ${ }^{107} \mathrm{In}$ this diagram each quark loop produces an effective $C$-odd vertex for electrostatic gluons 111 which in turn give rise to a logarithmically enhanced contribution at order $g^{6}$, reading 107

$$
\frac{\tilde{\chi}}{\chi_{0}} \simeq-\frac{\left(N^{2}-1\right)\left(N^{2}-4\right)}{128 N^{2}}\left(\frac{g}{\pi}\right)^{6} \log \frac{1}{g} .
$$

This vanishes in $\mathrm{SU}(2)$ gauge theory (though not in $\mathrm{QED}{ }^{107}$ ), and in (massless) QCD this leads to

$$
\left.\frac{\tilde{\chi}}{\chi_{0}}\right|_{N=3} \simeq-\frac{5}{144}\left(\frac{g}{\pi}\right)^{6} \log \frac{1}{g} \simeq-\frac{10}{9 \pi^{3}} \alpha_{s}^{3} \log \left(1 / \alpha_{s}\right) .
$$




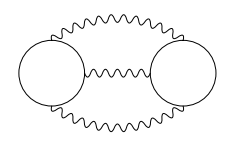

Fig. 21. Lowest-order diagram in the thermodynamic potential that contributes to offdiagonal susceptibilities $\partial \mathcal{N}_{i} / \partial \mu_{j}$ at $\mu=0$.

Assuming that the undetermined (though perturbatively computable) constant under the log is of order one, the natural order of magnitude of $\tilde{\chi} / \chi_{0}=\tilde{\chi} / T^{2}$ is $\sim 10^{-4}$ for $T \sim 3 T_{c}$. Recent lattice studies in $N_{f}=2$ QCD 101103 have found only values consistent with zero so far, with errors claimed to be below $10^{-6}$, but this perhaps remains to be confirmed by independent groups before it can be viewed as a new puzzle arising from the comparison of lattice and perturbation theory.

\section{Conclusion and perspectives}

In this paper, we have reviewed progress made recently in the calculation of the high temperature quark-gluon plasma using weak coupling techniques. Such calculations meet with difficulties, many of which are not typical of gauge theories, but also occur in scalar field theories. In fact, what determine the validity of a weak coupling expansion in thermal field theories is not only the strength of the coupling, but also the magnitude of the thermal fluctuations which varies according to the relevant momentum scales.

The thermodynamical functions are dominated by hard degrees of freedom, carrying momenta of the order of the temperature $T$. Soft modes, with typical momenta of order $g T$, contribute a correction, which however turns out to be difficult to calculate, for essentially two reasons. First, the dynamics of the soft modes receive contributions from the hard modes which can only be taken into account through all order resummations. Second, although the interactions among the soft modes can be treated perturbatively, the corresponding perturbation theory is an expansion in powers of $g$ rather than $g^{2}$, as it is for the hard modes. The latter feature is what causes the apparent poor convergence of perturbation theory which has been observed in calculations up to order $g^{5}$, both in the scalar case and in QCD.

In QCD, because of the unscreened magnetic fluctuations, perturbation theory eventually breaks down. But this occurs at order $g^{6}$ only, and accordingly one could expect the contribution of the magnetic fluctuations to 
the pressure to be numerically small. This is in fact suggested by detailed calculations based on dimensional reduction 63 . Therefore most of this review has concentrated on the difficulties which are common to all thermal field theories, and which involve the momentum scale $k \sim g T$.

The various resummation techniques which have been proposed to overcome the difficulties of perturbation theory rely to some degree on a separation of scale between hard and soft excitations, and follow the general pattern of effective theories. This is obviously the case for the approaches based on dimensional reduction, but is also true of screened perturbation theory and $\Phi$-derivable approximations, although somewhat more indirectly. In dealing with the soft sector, most resummations use the hard thermal loops as common building blocks. Non-linear "gap equations" for the calculation of "thermal mass" parameters emerge in various forms, and the difficulties associated with the ultraviolet renormalizations are dealt with in different fashions, depending on the specific approximations.

In "Screened Perturbation Theory", and its extension to QCD referred to as "HTL-perturbation theory", the use of the HTL Lagrangian as a basis for the resummation has the advantage to preserve manifest gauge symmetry, but also the drawback to lead to rather complex calculations beyond the lowest order. Besides the HTLs are used for all momenta, soft and hard, while they are accurate only at soft momenta. At any finite order in this scheme, one meets with temperature-dependent UV divergences whose renormalization requires the use of "thermal counterterms". Although they get in principle corrected gradually as one includes higher and higher orders, these features remain unsatisfactory in the low order calculations that one is able to perform.

At least at the level of the formalism, $\Phi$-derivable approximations provides a conceptually clearer perspective on the general problem of propagator renormalization than the simple "add and subtract" approach of SPT. The overcounting is automatically avoided by using skeleton expansions, and the gap equations emerge naturally, via the variational principle for the thermodynamic potential. The HTLs appear as lowest-order approximations to the solutions of the gap equations, and are used only in the kinematical domains where they are legitimate approximations. However, the practical implementation of $\Phi$ derivable approximations is not exempt of difficulties: In general, the gap equations are non-local, which complicates both their UV renormalization, and their numerical resolution. A further complication comes from the fact that the $\Phi$-derivable approximations violate gauge symmetry. (However, this violation is parametrically under 
control, which leaves the possibility to construct approximate solutions to the gap equations which are gauge-invariant.)

One of the most obvious advantages of $\Phi$-derivable approximations remains the simplifications they allow in the calculation of the entropy: The two-loop calculation for the pressure turns into an effectively one loop calculation for the entropy, which furthermore is finite once the self-energy involved in the calculation has been made finite. The results reported in Refs. ${ }^{232425}$ have been obtained however after additional approximations. In the scalar 2-loop model, which is exactly solvable, it has been possible to test these approximations by comparing against the respective exact results. In QCD, where such a comparison is not possible, further refinements should be studied, in particular to deal with non-local effects in the gap equations. Also, the calculation of the entropy is built on real-time information, namely the dispersion relation for the hard quasiparticles. Understanding the relation with purely Euclidean formalisms, such as that of dimensional reduction where this information does not enter in an explicit fashion, is certainly worth further investigations.

In closing, one should perhaps emphasize that most of the calculations reported in this review point to the validity of the quasiparticle picture of the quark-gluon plasma at high temperature. That is, they support the idea that, by suitably dressing the elementary degrees of freedom, one could minimize the effects of their residual interactions. This is particularly transparent in the entropy calculation. The results obtained in this manner for the thermodynamic potentials and also for quark susceptibilities seem to encourage this physical picture, which gives hope for the eventual applicability of correspondingly improved perturbation theory to dynamical quantities.

\section{Acknowledgments}

This work has been supported by the Austrian-French scientific exchange program Amadeus of APAPE and ÖAD, and the Austrian Science Foundation FWF, project no. P14632.

\section{References}

1. J. C. Collins and M. J. Perry, Phys. Rev. Lett. 34, 1353 (1975).

2. F. Karsch, Lect. Notes Phys. 583, 209 (2002).

3. E. Laermann and O. Philipsen, hep-ph/0303042.

4. R. D. Pisarski, Phys. Rev. D62, 111501 (2000); P. N. Meisinger, T. R. Miller and M. C. Ogilvie, Phys. Rev. D65, 034009 (2002). 
5. J. I. Kapusta, Finite-temperature field theory (Cambridge University Press, Cambridge, UK, 1989).

6. M. Le Bellac, Thermal Field Theory (Cambridge University Press, Cambridge, UK, 1996).

7. A. V. Smilga, Phys. Rept. 291, 1 (1997).

8. J.-P. Blaizot and E. Iancu, Phys. Rept. 359, 355 (2002).

9. E. Braaten and R. D. Pisarski, Nucl. Phys. B337, 569 (1990).

10. J. Frenkel and J. C. Taylor, Nucl. Phys. B334, 199 (1990).

11. J.-P. Blaizot, J.-Y. Ollitrault and E. Iancu, in "Quark-gluon plasma", ed. R. Hwa, vol. 2, pp. 135-210 (World Scientific, Singapore, 1995).

12. J.-P. Blaizot and E. Iancu, Nucl. Phys. B390, 589 (1993); Phys. Rev. Lett. 70, 3376 (1993).

13. J.-P. Blaizot and E. Iancu, Nucl. Phys. B417, 608 (1994).

14. V. P. Nair, Phys. Rev. D48, 3432 (1993); Phys. Rev. D50, 4201 (1994).

15. D. Bödeker, Phys. Lett. B426, 351 (1998); Nucl. Phys. B559, 502 (1999).

16. J.-P. Blaizot and E. Iancu, Nucl. Phys. B557, 183 (1999).

17. K. Kajantie, M. Laine, K. Rummukainen and Y. Schröder, Phys. Rev. Lett. 86, 10 (2001).

18. F. Karsch, A. Patkós and P. Petreczky, Phys. Lett. B401, 69 (1997).

19. S. Chiku and T. Hatsuda, Phys. Rev. D58, 076001 (1998).

20. J. O. Andersen, E. Braaten and M. Strickland, Phys. Rev. D63, 105008 (2001).

21. J. O. Andersen, E. Braaten and M. Strickland, Phys. Rev. Lett. 83, 2139 (1999); Phys. Rev. D61, 014017, 074016 (2000).

22. G. Baym, Phys. Rev. 127, 1391 (1962).

23. J.-P. Blaizot, E. Iancu and A. Rebhan, Phys. Rev. Lett. 83, 2906 (1999).

24. J.-P. Blaizot, E. Iancu and A. Rebhan, Phys. Lett. B470, 181 (1999).

25. J.-P. Blaizot, E. Iancu and A. Rebhan, Phys. Rev. D63, 065003 (2001).

26. B. Vanderheyden and G. Baym, J. Stat. Phys. 93, 843 (1998).

27. A. Peshier, B. Kämpfer, O. P. Pavlenko and G. Soff, Phys. Rev. D54, 2399 (1996).

28. P. Levai and U. Heinz, Phys. Rev. C57, 1879 (1998).

29. A. Peshier, B. Kämpfer and G. Soff, Phys. Rev. C61, 045203 (2000).

30. R. A. Schneider and W. Weise, Phys. Rev. C64, 055201 (2001).

31. B. Kastening, Phys. Rev. D56, 8107 (1997).

32. T. Hatsuda, Phys. Rev. D56, 8111 (1997).

33. G. Cvetič and R. Kögerler, Phys. Rev. D66, 105009 (2002).

34. R. R. Parwani, Phys. Rev. D63, 054014 (2001); ibid., D64, 025002 (2001).

35. P. Arnold and C.-X. Zhai, Phys. Rev. D50, 7603 (1994).

36. R. Parwani and H. Singh, Phys. Rev. D51, 4518 (1995).

37. E. Braaten and A. Nieto, Phys. Rev. D51, 6990 (1995).

38. R. Kobes, G. Kunstatter and A. Rebhan, Phys. Rev. Lett. 64, 2992 (1990); Nucl. Phys. B355, 1 (1991); A. Rebhan, Lect. Notes Phys. 583, 161 (2002).

39. A. K. Rebhan, Phys. Rev. D48, 3967 (1993). Nucl. Phys. B430, 319 (1994).

40. J.-P. Blaizot, E. Iancu and R. R. Parwani, Phys. Rev. D52, 2543 (1995).

41. P. Arnold and C.-X. Zhai, Phys. Rev. D51, 1906 (1995). 
42. C.-X. Zhai and B. Kastening, Phys. Rev. D52, 7232 (1995).

43. E. Braaten and A. Nieto, Phys. Rev. D53, 3421 (1996).

44. P. Ginsparg, Nucl. Phys. B170, 388 (1980).

45. T. Appelquist and R. D. Pisarski, Phys. Rev. D23, 2305 (1981).

46. S. Nadkarni, Phys. Rev. D27, 917 (1983).

47. S. Nadkarni, Phys. Rev. D38, 3287 (1988).

48. N. P. Landsman, Nucl. Phys. B322, 498 (1989).

49. E. Braaten, Phys. Rev. Lett. 74, 2164 (1995).

50. K. Kajantie, M. Laine, K. Rummukainen and M. Shaposhnikov, Nucl. Phys. B458, 90 (1996).

51. A. Rebhan, in "Strong and Electroweak Matter 2000", ed. C. P. Korthals Altes, pp. 199-203 (World Scientific, Singapore, 2000).

52. J. M. Luttinger and J. C. Ward, Phys. Rev. 118, 1417 (1960).

53. C. De Dominicis and P. C. Martin, J. Math. Phys. 5, 14, 31 (1964).

54. J. M. Cornwall, R. Jackiw and E. Tomboulis, Phys. Rev. D10, 2428 (1974).

55. E. Braaten and E. Petitgirard, Phys. Rev. D65, 041701, 085039 (2002).

56. H. van Hees and J. Knoll, Phys. Rev. D65, 025010, 105005 (2002); Phys. Rev. D66, 025028 (2002).

57. J.-P. Blaizot, E. Iancu and U. Reinosa, hep-ph/0301201.

58. I. T. Drummond, R. R. Horgan, P. V. Landshoff and A. Rebhan, Nucl. Phys. B524, 579 (1998).

59. J. O. Andersen, E. Braaten, E. Petitgirard and M. Strickland, Phys. Rev. D66, 085016 (2002).

60. J. O. Andersen, E. Petitgirard and M. Strickland, hep-ph/0302069.

61. E. Riedel, Z. Phys. 210, 403 (1968).

62. U. Kraemmer, A. K. Rebhan and H. Schulz, Ann. Phys. 238, 286 (1995).

63. K. Kajantie, M. Laine, K. Rummukainen and Y. Schröder, hep-ph/0211321.

64. T. van Ritbergen, J. A. M. Vermaseren and S. A. Larin, Phys. Lett. B400, 379 (1997).

65. G. Boyd et al., Nucl. Phys. B469, 419 (1996).

66. M. Okamoto et al., Phys. Rev. D60, 094510 (1999).

67. A. Papa, Nucl. Phys. B478, 335 (1996); B. Beinlich, F. Karsch, E. Laermann and A. Peikert, Eur. Phys. J. C6, 133 (1999).

68. F. Karsch, E. Laermann and A. Peikert, Phys. Lett. B478, 447 (2000).

69. A. Ali Khan et al., Phys. Rev. D64, 074510 (2001).

70. K. Kajantie, M. Laine, K. Rummukainen and M. E. Shaposhnikov, Nucl. Phys. B503, 357 (1997).

71. A. M. Polyakov, Phys. Lett. B72, 477 (1978).

72. A. D. Linde, Phys. Lett. B96, 289 (1980).

73. D. J. Gross, R. D. Pisarski and L. G. Yaffe, Rev. Mod. Phys. 53, 43 (1981).

74. J.-P. Blaizot, E. Iancu and A. Rebhan, hep-ph/0303045.

75. M. Laine, hep-ph/0301011.

76. E. Braaten and R. D. Pisarski, Phys. Rev. D45, 1827 (1992).

77. J. Frenkel and J. C. Taylor, Nucl. Phys. B374, 156 (1992).

78. R. Baier and K. Redlich, Phys. Rev. Lett. 84, 2100 (2000).

79. J. O. Andersen and M. Strickland, Phys. Rev. D64, 105012 (2001). 
80. B. A. Freedman and L. D. McLerran, Phys. Rev. D16, 1130 (1977).

81. A. Arrizabalaga and J. Smit, Phys. Rev. D66, 065014 (2002).

82. A. Peshier, Phys. Rev. D63, 105004 (2001).

83. O. K. Kalashnikov and V. V. Klimov, Sov. J. Nucl. Phys. 31, 699 (1980).

84. H. A. Weldon, Phys. Rev. D26, 1394 (1982).

85. R. D. Pisarski, Physica A158, 146 (1989).

86. V. V. Klimov, Sov. J. Nucl. Phys. 33, 934 (1981).

87. R. D. Pisarski, Nucl. Phys. A498, 423C (1989).

88. J.-P. Blaizot and J.-Y. Ollitrault, Phys. Rev. D48, 1390 (1993).

89. U. Kraemmer, M. Kreuzer and A. Rebhan, Ann. Phys. 201, 223 (1990).

90. F. Flechsig and A. K. Rebhan, Nucl. Phys. B464, 279 (1996).

91. H. Schulz, Nucl. Phys. B413, 353 (1994).

92. G. D. Moore, JHEP 0210, 055 (2002); A. Ipp, G. D. Moore and A. Rebhan, JHEP 0301, 037 (2003).

93. A. Rebhan, hep-ph/0301130.

94. F. Karsch, Nucl. Phys. Proc. Suppl. 83, 14 (2000).

95. I. T. Drummond, R. R. Horgan, P. V. Landshoff and A. Rebhan, Phys. Lett. B460, 197 (1999).

96. Z. Fodor and S. D. Katz, Phys. Lett. B534, 87 (2002); JHEP 0203, 014 (2002); P. de Forcrand and O. Philipsen, Nucl. Phys. B642, 290 (2002); C. R. Allton et al., Phys. Rev. D66, 074507 (2002).

97. Z. Fodor, S. D. Katz and K. K. Szabo, hep-lat/0208078.

98. K. K. Szabo and A. I. Toth, hep-ph/0302255.

99. P. Romatschke, hep-ph/0210331.

100. M. Asakawa, U. W. Heinz and B. Müller, Phys. Rev. Lett. 85, 2072 (2000); S. Jeon and V. Koch, Phys. Rev. Lett. 85, 2076 (2000); M. Prakash, R. Rapp, J. Wambach and I. Zahed, Phys. Rev. C65, 034906 (2002).

101. R. V. Gavai, S. Gupta and P. Majumdar, Phys. Rev. D65, 054506 (2002).

102. R. V. Gavai and S. Gupta, Phys. Rev. D65, 094515 (2002).

103. R. V. Gavai and S. Gupta, hep-lat/0211015.

104. C. Bernard et al., hep-lat/0209079.

105. A. Vuorinen, hep-ph/0212283.

106. T. Toimela, Int. J. Theor. Phys. 24, 901 (1985).

107. J.-P. Blaizot, E. Iancu and A. Rebhan, Phys. Lett. B523, 143 (2001).

108. R. V. Gavai and S. Gupta, Phys. Rev. D64, 074506 (2001).

109. P. Chakraborty, M. G. Mustafa and M. H. Thoma, Eur. Phys. J. C23, 591 (2002); hep-ph/0303009.

110. J.-P. Blaizot, E. Iancu and A. Rebhan, hep-ph/0206280.

111. C. P. Korthals-Altes, R. D. Pisarski and A. Sinkovics, Phys. Rev. D61, 056007 (2000); A. Hart, M. Laine and O. Philipsen, Nucl. Phys. B586, 443 (2000); D. Bödeker and M. Laine, JHEP 0109, 029 (2001). 